\title{
MODELS FOR SUB-ALFVÉNIC MAGNETODYNAMICS OF FUSION PLASMAS
}

\author{
F. L. WAELBROECK* \\ Institute for Fusion Studies, University of Texas, Austin, Texas 78712
}

Received September 16, 2010

Accepted for Publication October 15, 2010

The models describing macroscopic magnetic perturbations that evolve slowly compared to the Alfvén velocity are reviewed. The perturbations of interest include tearing modes, resistive interchange and ballooning modes, internal kink modes, resistive wall modes, and resonant magnetic perturbations. Two important features that distinguish the various models are their descriptions of parallel dynamics and of ion gyration. The evolution of macroscopic modes is generally characterized by resonances that result in the development of small scales. For processes involving magnetic reconnection, for example, all scales from the ion down to the electron Larmor radius are generated nonlinearly. The magnetohydrodynamic model assumes that the gradient lengths are always greater than the ion Larmor radius and thus is unable to properly describe the resonances. The drift models rely on a much more detailed description of the motion that enables them to capture many of the features of the short-scale phenomena, but they remain limited by their local description of the effects of gyration, and by their inability to describe the effects of wave-particle interactions in the parallel dynamics. These limitations are remedied by the gyrokinetic model, which provides a consistent, first-principles description of all the dynamics below the ion cyclotron frequency, but this model is computationally costly and its range of practical applicability remains to be established. Lastly, the gyrofluid models constitute a family of closures based on the moments of the gyrokinetic equations. These models offer an attractive compromise between fidelity and computational cost but have only recently begun to be applied to macroscopic evolution.

KEYWORDS: magnetic perturbations, Alfvén velocity, tearing modes, resistive interchange and ballooning modes, internal kink modes, resistive wall modes, drift model, gyrofluid model

Note: Some figures in this lecture are in color only in the electronic version.

\section{INTRODUCTION}

Macroscopic instabilities eject large fractions of the energy stored in fusion plasmas onto the plasma-facing components and thus are very dangerous. Controlling these instabilities is necessary for the success of fusion. ${ }^{1}$ The most useful tool for doing so has been the ideal magnetohydrodynamic (MHD) model, ${ }^{2}$ which plays a central role in guiding the design and operation of fusion devices. Remarkably, the MHD model provides quantitative predictions both for the amplitude of the response of stable plasmas to external perturbations, ${ }^{3}$ and for the waveforms and stability thresholds for the external kink ${ }^{4}$ and peeling-ballooning modes. ${ }^{5}$

\footnotetext{
*E-mail: flw@mail.utexas.edu
}

The MHD model is much less successful, however, in predicting the characteristic time scales for macroscopic plasma instabilities. The characteristic time $\tau_{A}$ for ideal MHD instabilities may be obtained from the balance between inertia and the kink force, $m_{i} n \partial \mathbf{V} / \partial t \sim$ $\mathbf{J} \times \mathbf{B}$, where $m_{i}$ is the ion mass, $n$ is the plasma density, $\mathbf{V}$ is the plasma velocity, $\mathbf{B}$ is the magnetic field, and $\mathbf{J}=$ $\nabla \times \mathbf{B} / \mu_{0}$ is the plasma current density. One finds

$$
\tau_{A}=L / V_{A},
$$

where

$$
\begin{aligned}
V_{A} & =B / \sqrt{\mu_{0} n m_{i}}=\text { Alfvén velocity } \\
L & =\text { dimension of the plasma } \\
B & =|\mathbf{B}|=\text { magnitude of the magnetic field. }
\end{aligned}
$$


The time $\tau_{A}$, called the Alfvén time, is the time it would take for the entire plasma to move out of its initial volume as a result of an ideal instability. Under normal conditions in fusion experiments, the characteristic growth time for macroscopic instabilities is much longer than the Alfvén time.

In an effort to describe more slowly growing modes, such as the internal kink, tearing modes, and resistive edge-localized modes (ELM), several linear ${ }^{6-8}$ and nonlinear ${ }^{9-13}$ codes solving the resistive MHD equations have been developed. These codes have enjoyed some successes in modeling the results of experiments with comparatively high collision frequency, ${ }^{14-17}$ but they have been largely unable to predict quantitatively the occurrence or evolution of long-wavelength, sub-Alfvénic instabilities in low-collisionality experiments. ${ }^{18,19}$ In particular, existing MHD codes are unable to predict the period of sawtooth oscillations, the repetition frequency of the ELM, and either the onset threshold or the saturation amplitude for the neoclassical tearing mode (NTM). The instabilities of interest share the following characteristic properties:

1. Sub-Alfvénic evolution. Their evolution takes place over a time $\tau$ that is intermediate between the Alfvén time $\tau_{A}$ and the confinement time $\tau_{E}\left(\tau_{A} \ll \tau \ll \tau_{E}\right)$. We will see that this circumstance causes resonances that are responsible for the second characteristic property.

2. Localized short-scale structures. Due to resonant phenomena and nonlinearity, long-wavelength modes exhibit localized, short-wavelength structures that play an important role in setting the rate of evolution of the mode (Fig. 1). Studies show that magnetic reconnection, in particular, generates singular current structures of width

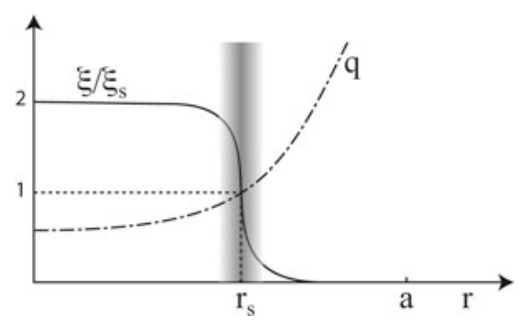

Fig. 1. Internal-kink wave function. The solid line represents the $m=n=1$ harmonic of the displacement $\xi$ associated with an internal kink mode, as a function of the minor radius $r$. The dashed-dotted line represents the safety factor $q$, which measures the winding ratio of the field lines on the toroidal flux surfaces. The wave function has macroscopic extent but exhibits singular behavior at the resonant surface $r_{s}$ where $q=m / n=1$. Note that the short-scale structure (the drop at $r=r_{s}$ ) is localized in the region where the flute ordering applies (shaded band). In this region the singularity is resolved by inertia.

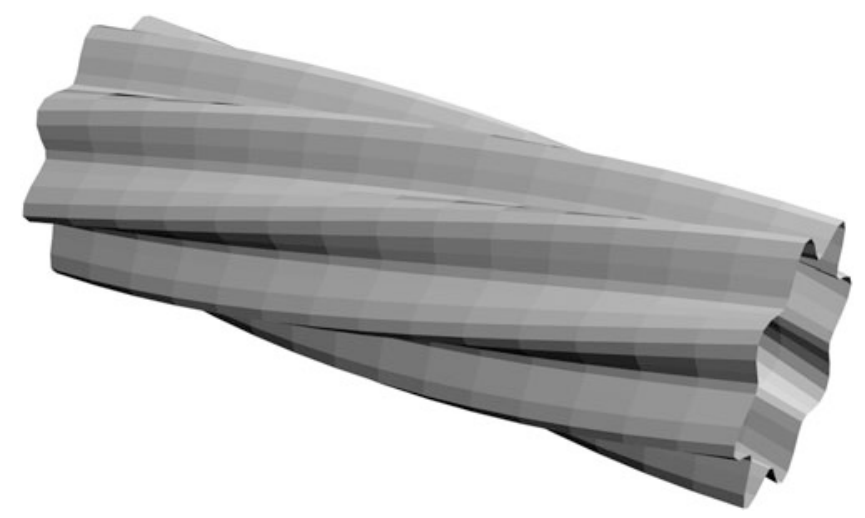

Fig. 2. Illustration of the distortion of a resonant magnetic surface, showing that near the resonant surface, the perturbation has a tendency to vary more rapidly in the direction perpendicular to the field than along the field. This tendency is described by the flute ordering $k_{\|} \ll k_{\perp}$.

less than the electron skin depth, $\lambda \ll d_{e}$, where $d_{e}=c /$ $\omega_{p e}$ is the electron skin depth and $\omega_{p e}$ is the electron plasma frequency. ${ }^{20}$

3. Failure of the flute ordering. Near the resonant layers, the perturbations tend to be highly elongated along the magnetic field, giving flux surfaces the appearance of grooved columns (Fig. 2). In the region where such geometric conditions prevail (see Fig. 1), the so-called flute ordering, $k_{\|} \ll k_{\perp}$, makes it possible to eliminate the compressional Alfvén dynamics. ${ }^{21}$ This is the basis for the reduced MHD model ${ }^{22,23}$ and several reduced twofluid models that offer the advantages of simplicity and physical transparency. ${ }^{24-26}$ In fusion experiments, unfortunately, $k_{\|} \sim \epsilon k_{\perp}$, where the inverse aspect ratio $\epsilon$ is typically no smaller than $1 / 3$ or so. Consequently, the flute ordering is generally considered inadequate for the global description of long-wavelength modes. It is often used, however, in local asymptotic investigations of the dynamics in the singular layers, especially those involving kinetic effects.

A consequence of these properties is that the evolution of sub-Alfvénic modes cannot be decoupled from the turbulence responsible for anomalous transport. The reason for this is that the scales characterizing the subAlfvénic singular structures, such as the ion Larmor radius, are generally indistinguishable from the scales of the turbulence. Thus, any model that offers a suitable description of the macroscopic modes near the resonant surfaces will also exhibit microscopic instabilities such as modes driven by the ion temperature gradient (ITG), trapped electrons (TEM), etc. ${ }^{27}$ The microscopic instabilities can sometimes be avoided by choosing parameters such that these modes are stable, for example, by neglecting temperature gradients and using large 
anomalous transport coefficients to damp the remaining drift instabilities, ${ }^{28-30}$ but this approach can be justified only in the presence of a scale separation between the turbulence and the resonant long-wavelength modes (e.g., for magnetic islands of width $W \gg \rho_{i}$ ).

The necessity of including the turbulent dynamics implies that the order of the system for any description of macroscopic sub-Alfvénic modes (i.e., the number of degrees of freedom, or the number of variables that have to be advanced in time) greatly exceeds that encountered in other domains of fusion theory. Until now, the order has generally been kept manageable by using fluid descriptions of the plasma, but this is unsatisfactory for several reasons. From the macroscopic point of view, fluid models fail to account for many phenomena, such as parallel heat flows, the effects of trapped particles, and neoclassical effects, that are important at the small collision frequencies that prevail in fusion experiments. From the microscopic point of view, the effect of collisionless kinetic damping (Landau damping) is known to suppress large-scale flows within the flux surfaces. These flows, which are generated nonlinearly by the turbulence, are called zonal flows by analogy with the east-west atmospheric flows along the earth's latitude lines. Existing fluid models either neglect Landau damping altogether, resulting in underestimation of the threshold and growth time for the instabilities, or they include a phenomenological model for Landau damping ${ }^{31}$ that overestimates the damping of the zonal flows ${ }^{32}$ and the amplitude of the turbulence. ${ }^{33}$

These observations have spurred efforts to develop kinetic models able to describe the evolution of macroscopic electromagnetic disturbances. In view of the very large order (i.e., number of degrees of freedom) of kinetic models, however, the problem is likely to remain challenging even for the next generation of computers.

In response to the difficulty of the problem, considerable efforts have been devoted to the development of models with reduced order. The methods for doing this can be separated into analytic methods (reduction) and numerical methods (discretization schemes and timestepping algorithms). Continued progress will clearly benefit from combining the insights associated with these two types of methods. The goal of the present lecture is to review the underlying ideas so as to provide a basis for future progress.

The primary consideration in developing models is the time and space scales of interest. We begin by discussing these in order to justify some of the properties listed above for the modes of interest.

\section{I.A. Time Scales}

Plasma displacements at the Alfvén velocity are adequately described by the MHD model. The conclusions from all the investigations of MHD dynamics is that Alfvénic instabilities result in violent plasma motions with nefarious consequences. In practice, however, macroscopic modes almost always grow slowly compared to the Alfvén time, for two reasons:

1. Slowness of control variables. The characteristic time for changing the equilibrium, which is fixed by a combination of plasma transport processes and externally controlled variables (such as heating, current drive, and shape control), greatly exceeds the Alfvén time. When a discharge crosses a stability threshold for a macroscopic mode, it will thus do so slowly compared to the nominal growth rate of the instability. In this respect the loss of stability is similar to that observed in the familiar drinking-bird toy (Fig. 3). For the drinking bird, the cooling of the head caused by evaporation of the liquid on the surface of its beak creates a vacuum that draws fluid from the bird's trunk into its head, thereby creating an inverted pendulum. The evaporation is slow compared to the nominal tipping time (the time for tipping if all the fluid were located in the head). As the center of gravity rises, the frequency of the pendulum slows, much like the frequency of precursor oscillations slow before a sawtooth crash in a tokamak. For small displacement, the angle of oscillation $\theta$ evolves according to

$$
\ddot{\theta}=\gamma_{0}^{2} \gamma_{m} t \theta,
$$

where the umlaut represents derivations with respect to time, $\gamma_{m}=\dot{m} / m$ is the rate at which mass rises from the trunk to the head, and $\gamma_{0}=\sqrt{2 g / \ell}$ is the nominal growth rate, with $g$ the acceleration of gravity and $\ell$ the length of the bird's neck. The solutions are Airy functions (Fig. 4) with argument $t / \tau$, where $\tau=\left(\gamma_{m} \gamma_{0}^{2}\right)^{-1 / 3}$ is a hybrid between the nominal time for the instability (in the case

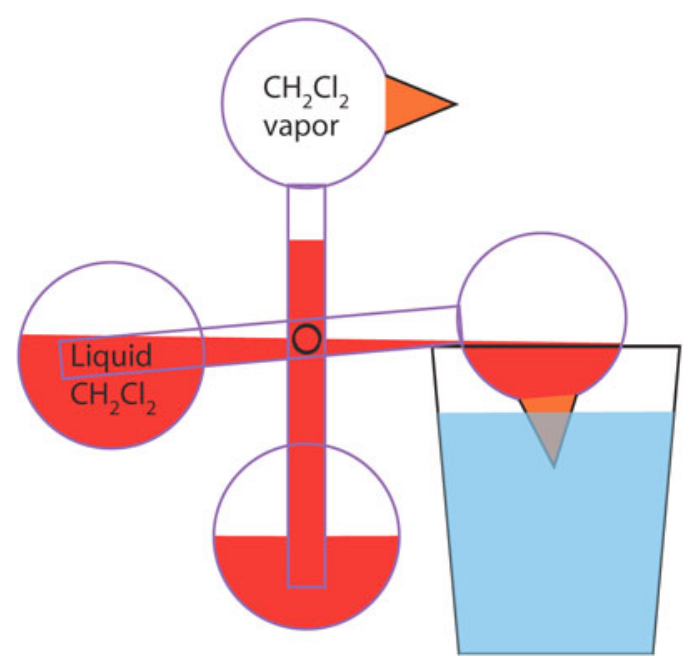

Fig. 3. The drinking bird toy illustrates the crossing of an ideal stability boundary (the inverted pendulum instability) by a slow transport process (the evaporation of the water on the bird's beak). 


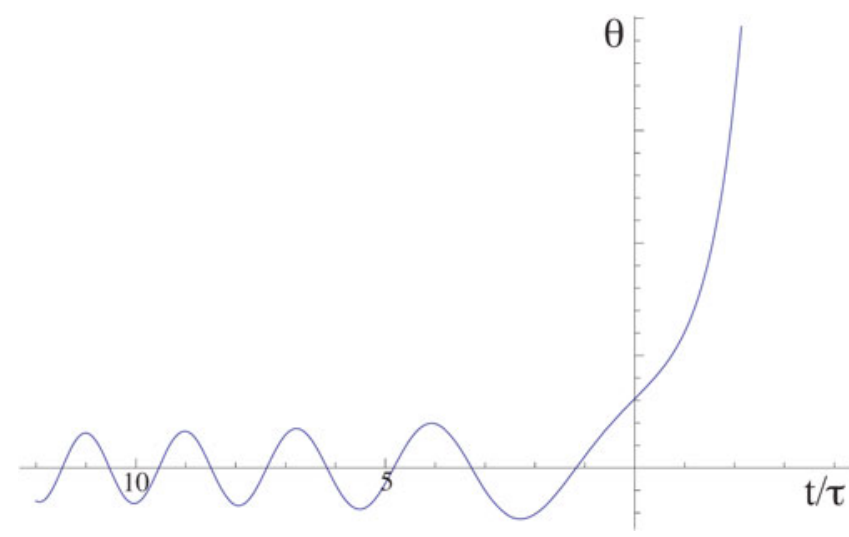

Fig. 4. Evolution of the displacement amplitude (in arbitrary units) for a system driven slowly through marginal stability, where $\tau$ is the hybrid growth time.

of the plasma, $\left.\tau_{A}\right)$ and the time describing the evolution of the equilibrium. ${ }^{34}$ Callen et al. have compared experimental observations of instabilities to the hybrid growth calculated under various assumptions concerning the nature of the underlying instability. ${ }^{35}$

2. Ideal saturation. For finite displacements, the inverted pendulum differs from ideal MHD instabilities in that crossing an ideal MHD stability threshold generally leads to the appearance of a nearby bifurcated equilibrium (Fig. 5). This constitutes the second reason for the slowness of MHD dynamics. Specifically, the frozen-in property of ideal MHD prevents the plasma from crossing magnetic flux surfaces. In ideal MHD, an equilibrium consisting of nested flux surfaces is thus topologically

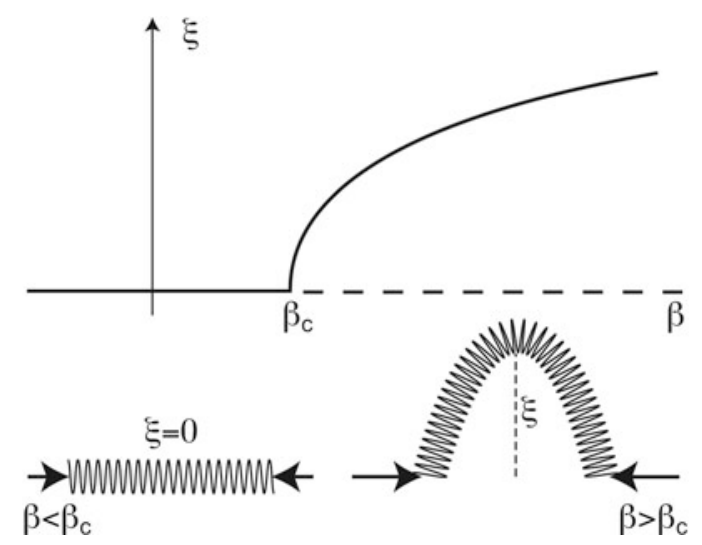

Fig. 5. Amplitude of the displacement (top) and shape (bottom) of a compressed spring undergoing an equilibrium bifurcation. As the compression parameter $\beta$ rises above the threshold $\beta_{c}$ determined by the stiffness of the spring, the system evolves to a bifurcated equilibrium with a displacement amplitude that scales like the square root of the transition parameter $\beta-\beta_{c}$. inalterable. As a result, both theory and simulations show that ideal instabilities saturate, and equilibria driven through a stability limit (by heating, for example) settle into new, bifurcated equilibria. ${ }^{36-38}$ Such bifurcated equilibria are sometimes observed in experiments, ${ }^{39-42}$ but more often, the presence of nonideal effects prevents saturation. The development of the instability beyond the ideal saturation amplitude is slowed to a time that is a hybrid between the Alfvén time and a time characterizing the dissipative processes (such as resistivity) responsible for the relaxation of the ideal MHD conservation properties. This is the case, in particular, for instabilities where the rate is controlled by magnetic reconnection, such as the internal kink mode. ${ }^{42}$ Another example is the resistive wall mode, an external kink instability for which the growth is fixed by the characteristic time for diffusion of the magnetic field through the conducting wall, called the wall time.

In conclusion, the macroscopic modes of interest in fusion experiments are characterized by velocities that are much smaller than the Alfvén velocity. This has important implications concerning the spatial behavior of the modes, which we now consider.

\section{I.B. Space Scales}

The slow evolution of the plasma implies that its state is never very far from equilibrium. Three-dimensional (3-D) equilibria, however, are known to exhibit singularities near resonant surfaces where the magnetic field lines close upon themselves. The reason for this is as follows.

The force-balance condition,

$$
\mathbf{J} \times \mathbf{B}=\nabla p,
$$

specifies the current density perpendicular to the magnetic field, $\mathbf{J}_{\perp}$, that must flow in order to provide the magnetic force that balances the gradient of the pressure $p$ :

$$
\mathbf{J}_{\perp}=\frac{\mathbf{b}}{B} \times \nabla p
$$

where $\mathbf{b}=\mathbf{B} / B$. This perpendicular current, however, has a nonvanishing divergence. In order to avoid charge buildup, the circuit must be closed by a parallel current, which is determined by the continuity equation, $\nabla \cdot \mathbf{J}=0$. In terms of the parallel current $J_{\|}=\mathbf{B} \cdot \mathbf{J} / B$, the continuity takes the form

$$
\begin{aligned}
\mathbf{B} \cdot \nabla\left(J_{\|} / B\right) & =-\nabla \cdot \mathbf{J}_{\perp} \\
& =-\nabla p \cdot \nabla \times(\mathbf{b} / B),
\end{aligned}
$$

where we have used Eq. (1) to eliminate $\mathbf{J}_{\perp}$. The pressure in Eq. (2), however, is itself affected by the distortions of the magnetic configurations. This can be seen by linearizing the parallel component of the equilibrium equation, 
$\underset{\widetilde{\mathbf{B}}}{\mathbf{B}} \cdot \nabla p=0$. Defining the perturbations by $\tilde{p}=p-p_{0}$ and $\widetilde{\mathbf{B}}=\mathbf{B}-\mathbf{B}_{0}$, where the 0 subscripts denote a reference axisymmetric state, we find

$$
\mathbf{B}_{0} \cdot \nabla \tilde{p}=-\widetilde{\mathbf{B}} \cdot \nabla p_{0} .
$$

Equations (2) and (3) are special cases of a common type of equation that takes the general form

$$
\mathbf{B} \cdot \nabla f=g,
$$

where $g$ is a known function of space. Such equations, called magnetic differential equations, play an important role in magnetic confinement theory. In a suitable magnetic coordinate system $(\psi, \theta, \zeta)$, where $\psi$ is the poloidal flux and $\theta$ and $\zeta$ are poloidal and toroidal angles, respectively, the solution takes the form

$$
f=i \frac{R q}{B_{\zeta}} \sum_{m, n} \frac{g_{m, n}}{m-n q} \exp (i m \theta+i n \zeta),
$$

where

$$
\begin{aligned}
q & =q(\psi)=\text { safety factor } \\
B_{\zeta} & =\text { toroidal component of the magnetic field } \\
R & =\text { major radius of the torus. }
\end{aligned}
$$

The $g_{m, n}$ are the Fourier coefficients of $g$,

$$
g=\sum_{m, n} \hat{g}_{m, n} \exp (i m \theta+i n \zeta) .
$$

The salient feature of the solution, Eq. (4), is its resonant denominator, $m-n q$. The presence of this denominator means that $f$ is singular on every rational surface $q=m / n$, unless the corresponding $g_{m, n}$ vanishes at that rational surface. We next examine how the resonance affects 3-D equilibria.

Applying the above solution to Eqs. (2) and (3), there follows

$$
J_{\|} / B=-i \frac{R q}{B_{\zeta}} \sum_{m, n} \frac{(\nabla p \cdot \nabla \times(\mathbf{b} / B))_{m, n}}{m-n q} \exp (i m \theta+i n \zeta)
$$

and

$$
\tilde{p}=-i \frac{R q}{B_{\zeta 0}} \frac{d p_{0}}{d \psi} \sum_{m, n} \frac{(\widetilde{\mathbf{B}} \cdot \nabla \psi)_{m, n}}{m-n q} \exp (i m \theta+i n \zeta) .
$$

Note that Eq. (5), unlike Eq. (6), applies to all orders in the perturbation amplitude, but we now linearize this equation in order to look at the effect of the pressure singularity on the parallel current. Taking the gradient of the pressure in Eq. (6) leads to terms proportional to $(d / d \psi)(m-n q)^{-1}=(m-n q)^{-2} n d q / d \psi$, further aggravating the singularity. We conclude that the degree of the current singularity is

$$
J_{\|} \sim(m-n q)^{-3}
$$

This shows that the resonances associated with closed field lines lead to serious, nonintegrable singularities on every rational surface. Physically, these singularities are caused by the inability of the parallel current to neutralize charge imbalances established across distinct closed field lines.

In order to place the above result in perspective, the following two considerations are relevant. First, the above simplified analysis is valid only for low values of $\beta=$ $2 \mu_{0} p / B^{2}$, the ratio of kinetic to magnetic pressure. For high- $\beta$ configurations, a more complicated analysis of the singularity, first carried out by Mercier, ${ }^{43}$ shows that the precise degree of the singularity in Eq. (7) depends on a geometric property called the magnetic well, which is associated with the curvature of the field lines. Second, we note that the argument leading to the result of Eq. (7) depends on the linearization of the equilibrium equation. As a result of nonlinear effects, the pressure may evolve so as to reduce the gradients on the low-order rational surfaces corresponding to the long-wavelength mode. This is the case, in particular, when the perturbation gives rise to isolated magnetic islands. ${ }^{44}$ In the presence of an island, the infinite number of closed field lines on the rational surface is replaced by a single closed field line, the magnetic axis of the island. Near the separatrix, the field lines weave across each other in a chaotic fashion, creating an ergodic layer where parallel currents can prevent the accumulation of charge caused by the diamagnetic drifts in Eq. (1), (Ref. 45), although the required currents may be large.

For an ideally unstable mode, the singularities at the resonant surfaces are resolved by inertia. As discussed above, however, MHD instabilities grow much more slowly than the Alfvén frequency, so that the width of the resonance can be very narrow for modes of interest in fusion. The width of the resonance may be estimated from the dispersion relation $\omega=k_{\|} V_{A}$ with $\omega=i \gamma$ and $k_{\|}$ depending on the distance from the rational surface through $k_{\|}=(m-n q) / R q$. The width of the resonance is thus $\delta \sim \gamma / V_{A}\left|\nabla k_{\|}\right|$. When $\gamma \ll \omega_{A}, \delta \ll r$, the minor radius.

In conclusion, resonant surface singularities in 3-D equilibrium configurations are an inescapable fact of life with far-reaching implications.

\section{I.C. Multiple Scale Methods}

It is commonplace to note that, as exhibited by the above discussion, the problem of describing the subAlfvénic evolution of MHD modes is characterized by multiple scales. A more interesting observation is that the dynamically important scales occupy a small fraction of the "scale space" $\omega-t$ and $\mathbf{k}-\mathbf{x}$. Specifically, rapid 
events are intermittent, and short-scale structures have localized extent in both space and scale. That is, $m-$ $n q \ll 1$ restricts both the radial variable $q$ and the scales $m$ and $n$. This creates uncountable opportunities for ingenious schemes aimed at reducing both the number of degrees of freedom and the time scales over which these degrees of freedom have to be tracked. The aim of this lecture is not only to describe some of these schemes but also to explain the general ideas used to devise them. The rapid evolution of computing technology means that the number of degrees of freedom that can be evolved is constantly growing, but at the cost of increasingly onerous restrictions on properties like memory and data locality. This results in a constantly evolving landscape for the set of computable models. There is thus a continuous need for new strategies enabling the optimal use of existing resources. It is hoped that the present review will provide a useful basis for researchers working on the development of such new strategies.

We begin by introducing some general and basic concepts in Sec. II. In particular, we introduce some of the ideas from dynamical systems theory that frame later discussions, and we compare and contrast the reduction technique with implicit algorithms. In Sec. III, we describe fluid closures with particular attention to the drift model, which is a two-fluid model that exploits the properties of sub-Alfvénic dynamics to achieve higher fidelity than generic two-fluid models. In Sec. IV, we present a brief description of the gyrokinetic model. In Sec. V, we describe the gyrofluid model, obtained by taking the moments of the gyrokinetic equations. Lastly, we summarize and discuss the results in Sec. VI.

\section{GENERAL CONSIDERATIONS}

\section{II.A. General Formulation}

It is often useful to think of the problem of predicting the evolution of the plasma in general dynamical terms. We denote the state of the plasma by the vector $\mathbf{X}$. In the real world, $\mathbf{X}$ has a countably infinite number of dimensions (when the fields are confined to a bounded volume, the countable nature of the dimension of $\mathbf{X}$ can be seen by expressing it in terms of a discrete Fourier series). When constructing a numerical model of the plasma, however, it is necessary to restrict $\mathbf{X}$ to a finite number, the value of which is limited by the memory of the computer.

We write the equation describing the evolution of $\mathbf{X}$ in the form

$$
\dot{\mathbf{X}}(t)=\mathbf{F}(\mathbf{X}(t), \mathbf{U}(t)),
$$

where $\mathbf{F}$ represents the forces acting on the system and $\mathbf{U}$ represents the input variables under the control of the experimentalist. We see that the order of the system of differential Eq. (8) is equal to the dimension of $\mathbf{X}$. We consider systems that are, in some sense, close to a reference state $\mathbf{X}_{0}(\mathbf{U})$ that is an equilibrium solution of the dynamical equation,

$$
\dot{\mathbf{X}}_{0}=\mathbf{F}\left(\mathbf{X}_{0}, \mathbf{U}\right)=0 .
$$

In general, the goal of the study is not merely to achieve the capability of obtaining solutions $\mathbf{X}(t)$ for any particular initial condition $\mathbf{X}(0)$ and set of input histories or "waveforms" $\mathbf{U}(t)$. Such a capability would certainly be useful for interpreting experiments and, if the model represents the experiment sufficiently faithfully, for predicting the results. It does little, however, to guide experimentalists in choosing the optimal input histories. A more ambitious goal is to gain knowledge and understanding of all the solutions and of how they depend on $\mathbf{U}$. That is, one would like to construct a map of the geometry of the solution curves to Eq. (8), rather than solve for a particular curve. The global understanding that such a map provides is necessary in order to guide the search for scenarios enabling the realization and sustainment of a desirable operation point.

A great deal of insight into the properties of the system can be gathered from the study of the linearized system obtained by expanding the force in a Taylor series about the equilibrium and retaining only the lowest-order terms. In terms of $\mathbf{x}=\mathbf{X}-\mathbf{X}_{0}$, the system takes the form

$$
\dot{\mathbf{x}}(t)=\mathbf{M x}(t),
$$

where

$$
\mathbf{M}=\left.\frac{\partial \mathbf{F}}{\partial \mathbf{X}}\right|_{\mathbf{x}_{0}}
$$

is an operator that takes the form of a matrix after discretization. An important feature of plasma systems is that they are generally nonnormal: that is,

$$
\mathbf{M}^{*} \mathbf{M} \neq \mathbf{M M}^{*},
$$

where $\mathbf{M}^{*}$ is the conjugate transpose of $\mathbf{M}$. A famous exception to this is ideal MHD, which is not only normal but Hermitian ${ }^{2}$ :

$$
\mathbf{M}_{\mathrm{MHD}}^{*}=\mathbf{M}_{\mathrm{MHD}} .
$$

In general, nonnormal $\mathbf{M}$ may give rise to strong amplification of applied perturbations even when the plasma is stable. ${ }^{46-50}$ It is also responsible for the fact that the eigenmodes of the resistive MHD equations do not converge to those of the ideal MHD system as the resistivity goes to zero. This is sometimes referred to as the Alfvén paradox. ${ }^{51}$

If the system has a complete set of distinct eigenvalues $s_{\mathbf{j}}$ and eigenvectors $\left|\mathbf{v}_{j}\right\rangle$ indexed by $\mathbf{j}=j_{1}, j_{2}, \ldots, j_{n}$, such that

$$
\mathbf{M}\left|\mathbf{v}_{\mathbf{j}}\right\rangle=s_{\mathbf{j}}\left|\mathbf{v}_{\mathbf{j}}\right\rangle,
$$


the general solution may be expressed in the form

$$
|\mathbf{x}(t)\rangle=\sum e^{s_{j} t}\left|\mathbf{v}_{j}\right\rangle\left\langle\mathbf{v}_{j} \mid \mathbf{x}_{0}\right\rangle .
$$

In general the eigenvalues $s_{\mathbf{j}}$ will span several orders of magnitude in frequency space. This is a nuisance, since the dynamics of interest usually involve a restricted set of modes with comparable frequencies or growth rates, while many high-frequency modes are damped or radiated out of the plasma and thus have small amplitudes. We next discuss two general schemes for dealing with this problem.

\section{II.B. Reduction versus Numerical Overstriding}

The preceding discussion shows that the problems we are interested in are characterized by multiple time and space scales. Many of these scales, however, are passive in the sense that the system rapidly reaches equilibrium at that scale and subsequently evolves slowly, adjusting continuously to changes in the parameters. In modeling this evolution one faces a fundamental choice:

1. Analytic reduction. Eliminate the fast time scale analytically and construct a model that describes only the slow dynamics. Two important examples of reduction that are used in every description of Alfvénic dynamics are the quasi-neutrality approximation, which eliminates space-charge oscillations, and the subluminal approximation, which eliminates electromagnetic radiation.

2. Numerical overstriding. Retain the fast dynamics but use a time-advance algorithm that allows the time step to exceed the short time scales. The most effective methods of doing this are with implicit ${ }^{52-57}$ and semi-implicit algorithms. ${ }^{10,58-61}$ Other related methods that have recently been the subject of interest are the projective integration and the heterogeneous multiscale methods. ${ }^{62,63}$

In order to explain and illustrate the above two approaches, we next consider the problem of maintaining the vertical position of the plasma. The analysis presented below borrows freely from the landmark papers by Lazarus and collaborators. ${ }^{64,65}$

Since the plasma inductance is independent of the vertical position, vertical control of the plasma provides a simpler example of a multiple time scale problem than horizontal control, which is inherently nonlinear. The fast time scale corresponds to the vertical oscillation of the plasma around its equilibrium position, at a frequency comparable to the Alfvén frequency. The slow time scale corresponds to the $L / R$ time for the dissipation of current fluctuations induced in the position control coils and the wall. In normal operation, vertical oscillations are damped, and the plasma resides very close to an equilibrium state at all times. As the parameters of the discharge (such as the plasma current and pressure) are varied, a feedback system changes the coil currents so as to maintain the plasma at the desired position. We next introduce a simple model for these dynamics. For the sake of clarity, we base the presentation on current filaments, but we note that models using distributed currents result in dynamical equations with the same form, the only changes being in the coefficients.

We consider a plasma represented by a filamentary current ring immersed in an axisymmetric magnetic field. The equation of motion for this ring is

$$
m_{p} \ddot{z}=-2 \pi X_{0} I_{p} B_{X}
$$

where

$$
\begin{aligned}
z= & \text { displacement of the ring from its equilibrium } \\
& \text { position at } z=0 \\
X_{0}= & \text { radius of the ring } \\
I_{p}= & \text { plasma current } \\
B_{X}= & \text { component of the magnetic field in the radial } \\
& \text { direction. }
\end{aligned}
$$

It can be shown a posteriori that the changes in the plasma current are negligible, so that we take $I_{p}$ to be constant. The magnetic field $B_{X}$ is created by external position control coils as well as by eddy currents $I_{v}$ flowing in the coils and vacuum vessel, which is treated as a part of the coil system for the purpose of the present stability analysis. We expand the magnetic field about the equilibrium position and use Biot-Savart's law to relate it to the external current ${ }^{64,65}$ :

$$
B_{X}=-\frac{I_{v}}{2 \pi X_{0}} \frac{\partial M_{v p}}{\partial z}+\frac{\mu_{0} I_{p} \Gamma n z}{4 \pi X_{0}^{2}} .
$$

Here,

$$
\Gamma=\frac{L_{e x t}}{\mu_{0} X_{0}}+\beta_{p}+\ell_{i} / 2+\frac{1}{2}
$$

where

$$
\begin{aligned}
L_{e x t}= & \text { external inductance of the plasma } \\
\ell_{i}= & \text { internal inductivity } \\
\beta_{p}= & \text { ratio of kinetic to poloidal magnetic energy } \\
M_{v p}= & \text { mutual inductance between the plasma and } \\
& \text { external currents. }
\end{aligned}
$$

The parameter $n$, defined by

$$
n=-\left.\frac{X_{0}}{B_{Z}} \frac{\partial B_{Z}}{\partial X}\right|_{X=X_{0}},
$$

is called the stability index for reasons that will soon become clear. Substituting $B_{X}$ in the equation of motion yields

$$
m_{p} \ddot{z}=\frac{\partial M_{v p}}{\partial z} I_{p} I_{v}-\frac{\mu_{0} I_{p}^{2} \Gamma n}{2 X_{0}} z
$$




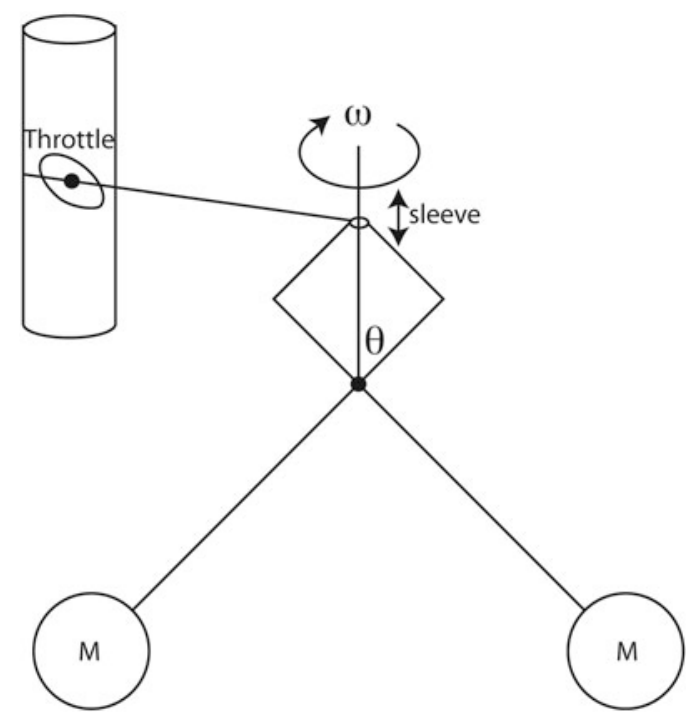

Fig. 6. Schematic of the Watt governor, a mechanical model for the system formed by the plasma and external conductors. In the governor, the spinning flyballs $M$ act on the throttle of a steam engine, which acts back on the flyballs through the rotation rate of the axis.

The system is closed with the circuit equation for the external current,

$$
\dot{I}_{v}+\gamma_{v} I_{v}+\frac{M_{v p}^{\prime} I_{p}}{L_{v}} \dot{z}=0
$$

where $\gamma_{v}$ is the decay rate for the external currents, $L_{v}$ is the self-inductance of the external conductors, and the prime denotes differentiation with respect to $z$. Equations (11) and (12) represent a third-order linear system describing the dynamics of the system formed by the plasma and external conductors. These equations have the form of Eq. (9) with $\mathbf{x}=\left\{z, \dot{z}, M_{v p}^{\prime} I_{v} I_{p} / m_{p}\right\}$ and

$$
\mathbf{M}=\left(\begin{array}{ccc}
0 & 1 & 0 \\
-n \omega_{1}^{2} & 0 & 1 \\
0 & -n_{c} \omega_{1}^{2} & -\gamma_{v}
\end{array}\right)
$$

where $\omega_{1}^{2}=\mu_{0} I_{p}^{2} \Gamma / 2 m_{p} X_{0}$ is a characteristic frequency for the vertical oscillation of the plasma in the stable regime, and $n_{c}=2\left(M_{v p}^{\prime}\right)^{2} X_{0} / \mu_{0} \Gamma L_{v}$ measures the passive stabilization of the plasma by the external currents. It is interesting to note that the above model for the plasma and conductors takes the same form as the equations derived by Watt to describe the centrifugal governor, one of the first mechanical feedback systems to be investigated for its stability in an attempt to prevent the problem of "hunting," the oscillation of the system about its desired operating point. ${ }^{66}$ The governor may thus be seen as a mechanical analogy for the tokamak, the flyballs play-
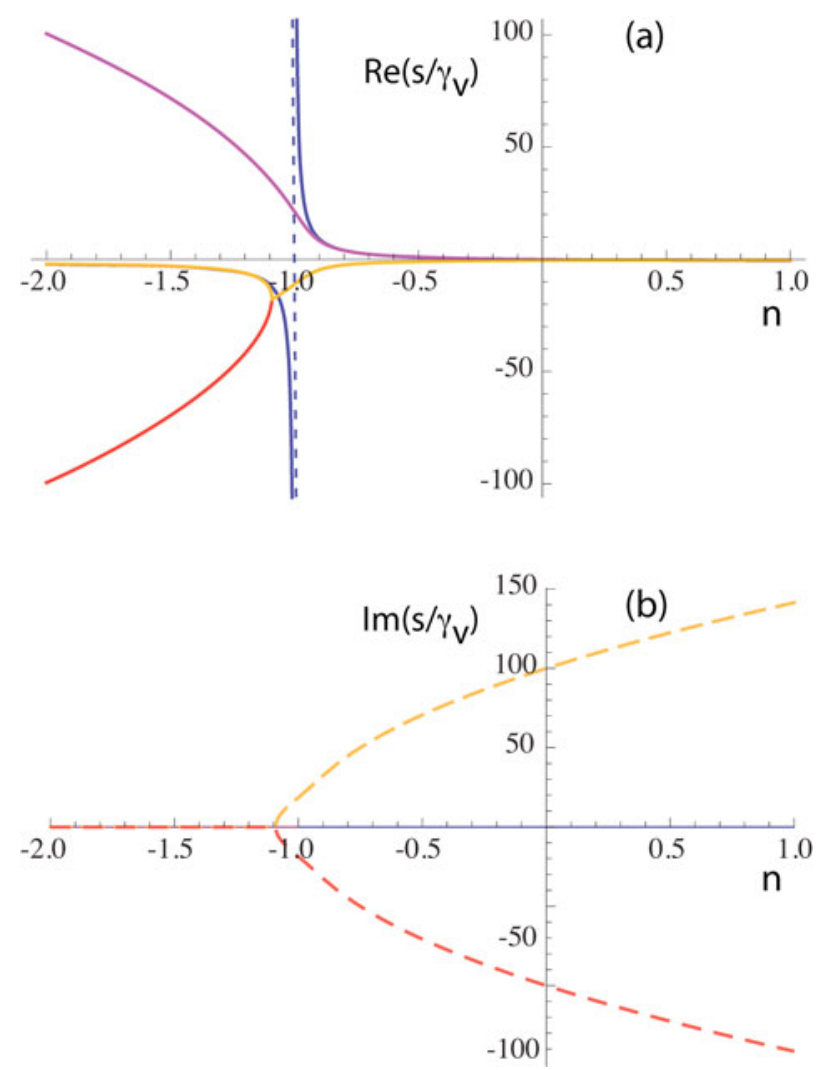

Fig. 7. Roots of the dispersion relation for the vertical stability problem as a function of the stability index $n$ : (a) growth rate and (b) frequency of the three roots, normalized to the wall time $\gamma_{v}^{-1}$, for $\omega_{1} / \gamma_{v}=100$ and $n_{c}=1$. In (a), the hyperbola with asymptote (dashed line) at $n=-1$ shows the growth rate calculated with the reduced model and given in Eq. (16).

ing the role of the plasma and the steam engine playing the role of the vacuum vessel (Fig. 6).

The evolution can be analyzed by taking the Laplace transform. The dispersion relation, or the solubility condition for the eigenmode Eq. (10), is a cubic polynomial in $s$ :

$$
\left(s^{2}+n \omega_{1}^{2}\right)\left(s+\gamma_{v}\right)+s \omega_{1}^{2} n_{c}=0 .
$$

The roots of the dispersion relation are plotted in Fig. 7. Note that to improve readability, the parameter $\omega_{1} / \gamma_{v}$ in Fig. 7 is smaller than the values encountered in experiments. In DIII-D, for example, $\omega_{1} / \gamma_{v} \sim 4 \times 10^{3}$.

In the limiting case where the coupling between the plasma and external conductors can be neglected, $n_{c}=0$, the roots are $s= \pm \omega_{1} \sqrt{-n}$ and $s=-\gamma_{v}$. The first pair of roots corresponds to the vertical motion of the plasma, and we see that for $n<0$ the plasma is unstable. The growth time is of the order of the Alfvén time, typically a microsecond or less. For $n>0$, perturbing the equilibrium leads to oscillation at a frequency comparable to the 
Alfvén frequency. This motivates the designation of $n$ as a stability index. Note that a similar analysis for the horizontal motion shows that in that case, stability requires $n<3 / 2$. The third and last root of Eq. (14), $s=-\gamma_{v}$, corresponds to the damping of currents induced in the conducting structures. A typical decay time for the vessel currents is a few milliseconds.

The large disparity between the characteristic time for the plasma motion and the current decay time, $\omega_{1} \gg$ $\gamma_{v}$, enables the behavior of the roots of the dispersion relation to be studied analytically. One finds that for $n_{c} \neq$ 0 , the marginal stability point at $n=0$ is moved to $n=$ $-n_{c}$. For $n<-n_{c}$, an Alfvénic instability develops. For $n>-n_{c}$, by contrast, we find a pair of lightly damped modes oscillating at the Alfvén frequency. That is, the conducting vessel stabilizes the Alfvénic instability in the parameter domain $-n_{c}<n<0$. In addition to the pair of Alfvénic roots, however, there is a third root describing a slow mode with a rate of evolution that is comparable to the inverse wall time, $\gamma_{v}$. The relatively slow growth of this mode makes it susceptible to stabilization by a feedback system. In order to do this, however, it is necessary to characterize its dynamics. We next present the analytic reduction method that provides a simple description for the slow mode.

\section{II.B.1. Analytic Reduction}

The reduction approach consists of two steps. In the first step, one eliminates analytically the fast time scale by taking the limit of $m_{p} \rightarrow 0$, corresponding to infinite Alfvén time. In this limit, $B_{X}=0$ must be satisfied so that

$$
z=\frac{2 M_{v p}^{\prime} X_{0}}{\mu_{0} I_{p} \Gamma n} I_{v} .
$$

This value of $z$ represents the instantaneous "equilibrium" solution, in the sense that it is the solution that satisfies force balance on the Alfvén time scale for that particular value of $I_{v}$.

The second step consists of substituting the equilibrium solution in the external circuit equation to obtain an equation describing the slow dynamics. We find

$$
\left(1+n_{c} / n\right) \dot{I}_{v}+\gamma_{v} I_{v}=0
$$

The eigenvalue is

$$
\gamma_{0}=-\gamma_{v} /\left(1+n_{c} / n\right) .
$$

Away from marginal stability, this value can be seen to be an excellent approximation to the exact root (Fig. 7).

The above procedure is extremely general and underlies almost every practical analysis of plasma evolution. As previously noted, the most frequently used reduction consists of eliminating the space-charge oscillations and light waves using a similar procedure. In particular, several fast electron waves, such as electron cyclotron oscillations, can be eliminated by taking the limit of vanishing electron mass $m_{e}$ in much the same way that we took $m_{p} \rightarrow 0$ above.

Unfortunately, even when clear separation of scales exists, it is sometimes impractical or impossible to carry out an analytical reduction. A common obstacle is the occurrence of spatial or temporal resonances, such that the scale separation breaks down locally. The situation may be further complicated by nonlinearity, such as cascade processes, that may lead to a breakdown in scale separation. In such cases, a numerical approach to the problem of multiple scales may be preferable. We next consider the most important of these approaches.

\section{II.B.2. Implicit Algorithm}

The problem with the direct numerical solution of Eqs. (11) and (12) is the need to adopt time steps shorter than the Alfvén time for accuracy as well as stability. Since we know that the fast oscillations are damped, we would like to find a stepping method that allows the integration to proceed with time steps comparable to the wall time, $\gamma_{v}^{-1}$. Implicit differencing provides such a method.

We introduce a discretization of time according to $\mathbf{x}^{j}=\mathbf{x}\left(t_{0}+j h\right)$, where $h$ is the time step and $j=$ $0,1,2, \ldots, T / h$, where $T$ is the final integration time. A general differencing algorithm for Eq. (8) is

$$
\mathbf{x}^{j+1}=\mathbf{x}^{j}+h\left[(1-\theta) F\left(\mathbf{x}^{j}\right)+\theta F\left(\mathbf{x}^{j+1}\right)\right] .
$$

For $\theta=0$, the force operator is evaluated at the old time step and the algorithm is explicit, whereas for $\theta=\frac{1}{2}$ we have a second-order accurate trapezoidal scheme. For $\theta=1$, lastly, the force is evaluated at the new time step and the algorithm is fully implicit. The difficulty of the above algorithm is that for $\theta>0$, it can be difficult to determine the new state $\mathbf{x}^{j+1}$. In the fully implicit case, for example, it is necessary to solve the equation

$$
\mathbf{x}^{j+1}-h F\left(\mathbf{x}^{j+1}\right)=\mathbf{x}^{j}
$$

for $\mathbf{x}^{j+1}$. In general, this is a nonlinear equation and its solution can be very demanding. The most straightforward method for inverting Eq. (17) is to use Newton's algorithm, but this requires both calculating and storing the Jacobian of $\mathbf{F}$, a generally prohibitively large matrix. Methods that avoid this have been developed and applied to MHD models ${ }^{53}$ and two-fluid models. ${ }^{54}$

It is instructive to consider the solution of the implicit algorithm for our simple vertical stability problem. In that case $\mathbf{F}(\mathbf{x})=\mathbf{M x}$ is linear and the solution is

$$
\mathbf{x}^{j+1}=(\mathbf{I}-h \mathbf{M})^{-1} \mathbf{x}^{j},
$$

where $\mathbf{I}$ is the unit matrix. Stepping forward until the system has advanced a time $t$, we find

$$
\mathbf{x}(t)=(\mathbf{I}-h \mathbf{M})^{-t / h} \mathbf{x}(0) .
$$


The evolution described by the above result becomes more transparent when expressed in terms of a basis formed of the eigenvectors of $\mathbf{M}$. If $\mathbf{T}$ is the matrix for the transformation to the eigenvector basis, $\mathbf{M}$ can be written as

$$
\mathbf{M}=\mathbf{T}^{-1} \mathbf{S T},
$$

where $\mathbf{S}$ is the diagonal matrix, with elements $S_{i j}=s_{j} \delta_{i, j}$, formed by the eigenvalues of M. Using T, Eq. (18) takes the form

$$
\mathbf{x}(t)=\mathbf{T}^{-1}(\mathbf{I}-h \mathbf{S})^{-t / h} \mathbf{T} \mathbf{x}(0),
$$

where the diagonal matrix in the middle of the right side is easily inverted and raised to the power of $t / h$, the number of time steps. The transformation matrices $\mathbf{T}$ may be ignored if one focuses on the evolution of the eigenvectors.

The explicit advance, by comparison, yields

$$
\mathbf{x}(t)=\mathbf{T}^{-1}(\mathbf{I}+h \mathbf{S})^{t / h} \mathbf{T} \mathbf{x}(0)
$$

The implicit and explicit advances may be compared to the exact solution,

$$
\mathbf{x}(t)=\mathbf{T}^{-1} \exp (\mathbf{S} t) \mathbf{T} \mathbf{x}(0) .
$$

Clearly, in the limit $h \rightarrow 0$, these expressions are all equivalent. Inspection of Eq. (19), however, shows that for $\left|h s_{ \pm}\right| \gg 1$, where the $s_{ \pm}= \pm i \omega_{1} \sqrt{n+n_{c}}$ are the fastest eigenvalues of $\mathbf{M}$, the oscillating modes evolve according to

$$
\mathbf{x}(t) \sim \exp \left[\left(\mp i \frac{\pi}{2}-\log \left(h \omega_{1} \sqrt{n+n_{c}}\right)\right) \frac{t}{h}\right] \mathbf{x}_{ \pm},
$$

where $\mathbf{x}_{ \pm}$are the eigenvectors corresponding to the two largest eigenvalues of $\mathbf{M}$. That is, the Alfvénic oscillations are now damped, and their oscillation frequency has been slowed to a value comparable with the time step $h$. Even for large values of $h \omega_{1}$, the logarithm term in Eq. (20) is usually not much larger than unity, so that the fast modes will have a half-life comparable to the time step. If the time step is chosen such that $\gamma_{v} h \ll 1$, the implicit scheme will describe the slow dynamics accurately. By contrast, it is easy to see that the explicit scheme results in the unphysical growth of the fast modes on the scale of the time step. A comparison of the implicit solution with the exact solution in the case of a stable plasma $\left(n / n_{c}=0.2>0\right)$ is shown in Fig. 8 . We see that the Alfvénic oscillations are damped in the first time step, and the implicit solution thereafter tracks the longtime average of the plasma position while taking steps that greatly exceed the vertical oscillation frequency.

It is important to note that for 3-D MHD models that aim to describe the dynamics of shear Alfvén modes

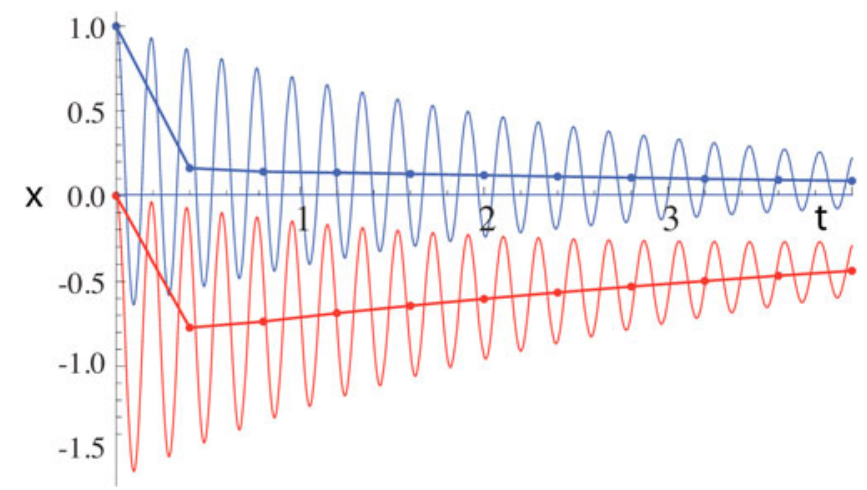

Fig. 8. Evolution of the vertical position (top two lines) and of the vessel current (bottom two lines) for $n=0.2, n_{c}=$ 1 , and $\omega_{1} / \gamma_{v}=30.0$. The continuous curves represent the exact solution and the dots joined by straight segments represent the implicit numerical solution with $h \omega_{1}=12.0$.

accurately while treating the compressional wave implicitly, numerical damping such as that found above is unacceptable. Fortunately, it is possible to devise methods that suppress the compressional wave through the use of numerically enhanced dispersion, rather than dissipation. ${ }^{58,59,67,68}$ These methods are essential to the ability of nonlinear MHD codes to model such slow processes as magnetic island growth. ${ }^{56,69,70}$

The advantage of the method of implicit advance becomes clear when considering a case where the plasma approaches the ideal vertical stability threshold, $n=-n_{c}$. In that case, the system evolves through a sequence of states such that the frequency of the fast oscillation mode, the vertical oscillation, becomes comparable to the wall time as the plasma approaches marginal stability. Near marginal stability, the reduction method becomes invalid due to the failure of the separation of time scales. The breakdown of the reduced model is displayed by the singularity of the growth rate calculated with this model, corresponding to the hyperbola in Fig. 7. The implicit method, by contrast, offers greater flexibility by describing the slow dynamics correctly, irrespective of whether the system is near marginal stability. Figure 9 shows the evolution of the vertical position for two different initial conditions for a plasma near the critical stability index, with $n / n_{c}=-0.9$ and $\omega_{1} / \gamma_{v}=100$. For these conditions the plasma is unstable, with a growth rate comparable to the wall time. This is near the limit of the growth rates that can be stabilized with a feedback system. We see that the implicit method gives an adequate description of the evolution for time steps that exceed the Alfvén time, $h \omega_{1}=2.0$.

The price paid for the flexibility of the implicit method is that the dimensionality of the model, or the order of the system of differential equations, is a factor of 3 times 


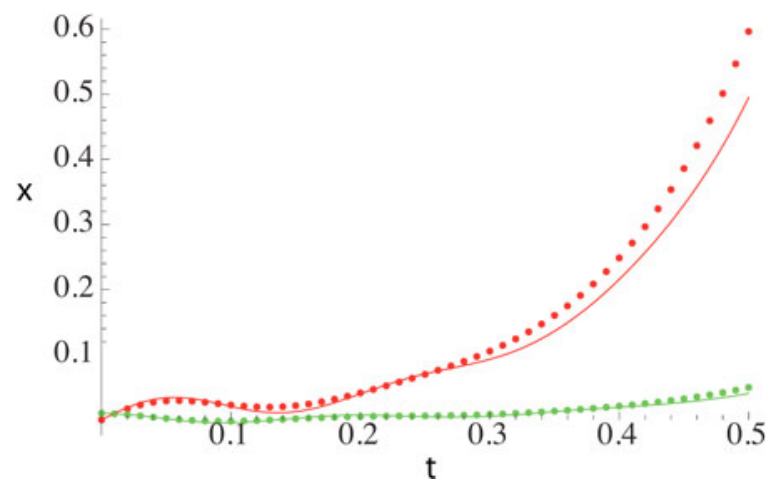

Fig. 9. Evolution of the vertical position for the fastest- and slowest-growing modes for $n=-0.9, n_{c}=1$, and $\omega_{1} /$ $\gamma_{v}=100.0$. The time step is $h \omega_{1}=1$.

higher than that for the reduced model. For more complex continuum models, such as MHD, the multiplier is generally smaller. Of greater concern is the cost for nonlinear systems of the inversion in Eq. (17). This cost can be avoided with semi-implicit methods, in which only an operator approximating the dominant linear part of the force $\mathbf{F}(\mathbf{X})$ is treated implicitly. Several fusion-MHD codes have adopted this strategy. ${ }^{9,13,71}$

In conclusion, we see that the implicit and semiimplicit methods constitute flexible techniques for integrating multiple-time-scale problems in cases where the separation of scales may disappear during the simulation. The reduction method, by contrast, requires strict scale separation for its validity but leads to a system of reduced order. Despite its limitations, the reduction approach is conceptually important for its ability to shed light on the geometry of the solutions to the dynamical equation.

\section{FLUID MODELS}

The main task of fusion theory is to construct simplified descriptions of the Maxwell-Boltzmann equations that describe the evolution of the low-density, high-temperature plasmas used in experiments. By simplified description, we mean either models of reduced order (that is, with fewer variables) or models that eliminate some of the time scales through an implicit treatment. In order to successfully predict the phenomena described in Sec. I, a combination of both of these approaches is necessary. The most useful techniques for achieving such simplification make use of the moment hierarchy. The principal application of the moment hierarchy is in the derivation of fluid models, and in the present lecture we restrict consideration to this application. We note, however, that the moment hierarchy also has applications in kinetic theory, both to simplify the analytic solution of the kinetic equations in transport theory ${ }^{21}$ and to construct implicit algorithms for particle-in-cell (PIC) codes. ${ }^{72,73}$

\section{III.A. The Moment Hierarchy}

The moment hierarchy corresponds to the sequence of equations obtained by taking successively higherorder moments of the Boltzman equation,

$$
\frac{\partial f_{s}}{\partial t}+\mathbf{v} \cdot \nabla f_{s}+\frac{e_{s}}{m_{s}}(\mathbf{E}+\mathbf{v} \times \mathbf{B}) \cdot \frac{\partial f_{s}}{\partial \mathbf{v}}=C_{s}(f),
$$

where

$$
\begin{aligned}
f_{s} & =\text { distribution function for species } s \\
e_{s}, m_{s} & =\text { species charge and mass } \\
C_{s} & =\text { collision operator. }
\end{aligned}
$$

Multiplying this equation $k$ times by $\mathbf{v}$ and integrating yields the $k$ 'th-order moment equation,

$$
\begin{gathered}
\frac{\partial \mathbf{M}_{s}^{(k)}}{\partial t}+\nabla \cdot \mathbf{M}_{s}^{(k+1)}+\frac{e_{s}}{m_{s}} \llbracket \mathbf{E M}_{s}^{(k-1)} \rrbracket \\
+\omega_{c s} \llbracket \mathbf{b} \times \mathbf{M}_{s}^{(k)} \rrbracket=\mathbf{C}^{(k)}
\end{gathered}
$$

where $\omega_{c s}=e_{s} B / m_{s}$ is the cyclotron frequency for species $s$. Here, the $k$ 'th-order moment of the distribution function is defined by

$$
\mathbf{M}_{s}^{(k)}(\mathbf{x}, t)=\int d^{3} v f_{s}(\mathbf{x}, \mathbf{v}, t) \mathbf{v} \ldots \mathbf{v}
$$

where $\mathbf{v} . . \mathbf{v}$ denotes $k$ factors of $\mathbf{v}$, and where the brackets $\llbracket \cdot \rrbracket$ indicate the sum over the cyclic permutations of uncontracted indices. Thus, $\mathbf{M}^{(k)}$ is in general a tensor of rank $k$, although it is often contracted to lower rank. An important characteristic of the moments is that highorder moments weigh more heavily the contributions from the high-velocity part of the distribution function, which corresponds to the part of phase space where the distribution is smallest. This makes high-order moments more susceptible to numerical noise in computation.

The most often stated property of the moment hierarchy is that each successive equation introduces a higherorder moment, so that the chain must be closed, either by appealing to some known features of the kinetic solution or simply by truncation. A somewhat less widely appreciated property is that for low-frequency evolution, in the sense that $\partial \mathbf{M} / \partial t \ll \omega_{c s} \mathbf{M}$, the moment hierarchy can be partially solved for the high-order moments in terms of the lower-order moments. This is the basis for all the magnetized fluid models, including MHD and the drift model. Before describing these models, we describe the reduction of the fluid equations that can be achieved under the assumptions $\omega \ll \omega_{p e}$ and $v_{p h}=\omega / k \ll c$, where $v_{p h}$ is the phase velocity of the waves of interest and $c$ is the speed of light. 


\section{III.B. The Quasi-Neutral and Subluminal Approximations}

Maxwell's equation,

$$
\frac{\partial \mathbf{E}}{\partial t}=c^{2}\left(\nabla \times \mathbf{B}-\mu_{0} \mathbf{J}\right),
$$

may be viewed as combining an equation for the two parts of the electric field: the longitudinal $(\nabla \cdot \mathbf{E})$ and the transverse or solenoidal $(\nabla \times \mathbf{E})$ parts. We consider each part in turn.

The longitudinal part of Maxwell's equation, obtained by applying the divergence operator to Eq. (24), is well known to be identical to the charge continuity equation,

$$
\frac{\partial \rho}{\partial t}=-\nabla \cdot \mathbf{J} .
$$

In a plasma, charge imbalance leads to extremely rapid oscillations that do not influence the slow evolution that is the subject of this lecture. It is interesting to estimate the charge imbalance under the assumption that the plasma velocity is no greater than the Alfvén velocity: $e\left(n_{i}-n_{e}\right)=\epsilon_{0} \nabla \cdot \mathbf{E} \sim \epsilon_{0} \nabla\left(V_{A} B\right)$. This leads to

$$
\left(n_{i}-n_{e}\right) / n \sim\left(V_{A} / c\right)^{2}\left(d_{i} / L\right),
$$

where $d_{i}=c / \omega_{p i}=\rho_{i} / \beta_{i}^{1 / 2}$ is the ion skin depth. As a result, almost all models for low-frequency plasma evolution assume quasi-neutrality. That is, they neglect the relative difference in the particle density. The adoption of the quasi-neutrality approximation amounts to a renunciation of the task of evolving the space charge as a dynamical variable. Consistency with the continuity equation requires that the constraint $\nabla \cdot \mathbf{J}=0$ be satisfied. This constraint may thus be thought of as the equilibrium condition for space-charge oscillations, where "equilibrium" is to be understood in the sense that $\omega \ll \omega_{p e}$. In principle, it is possible to use the equilibrium condition in conjunction with the momentum equations to calculate the value of $\nabla \cdot \mathbf{E}$, which can no longer be determined from Poisson's equation. This procedure is cumbersome, however, and we avoid it by using the quasi-neutrality approximation in conjunction with another approximation that we refer to as the subluminal approximation, because it relies on the smallness, compared to the speed of light, of the phase velocity of the waves under consideration.

The subluminal approximation is motivated by the observation that the right side of the solenoidal part of Maxwell's equation becomes large for $c / v_{p h} \rightarrow \infty$ :

$$
\frac{\partial}{\partial t} \nabla \times \mathbf{E}=c^{2}\left(\nabla^{2} \mathbf{B}-\mu_{0} \nabla \times \mathbf{J}\right) .
$$

For slow dynamics, it is thus necessary for the current to satisfy

$$
\nabla \times \mathbf{J}=\nabla^{2} \mathbf{B} / \mu_{0} .
$$

Again we find a cumbersome constraint on the current. In the case of the subluminal approximation, the constraint placed by the equilibrium Eq. (26) must be used to eliminate the solenoidal part of $\mathbf{E}$. Note that the subluminal approximation is often identified with the Darwin approximation, although the latter retains relativistic corrections to the particle motion that are neglected here.

We see from the above discussion that when taken together, the quasi-neutrality and subluminal approximations specify both $\nabla \cdot \mathbf{J}$ and $\nabla \times \mathbf{J}$, thereby eliminating the need to solve for the longitudinal and transverse parts of E separately. We may then use the electron momentum equation,

$$
\begin{aligned}
m_{e} n \frac{d \mathbf{V}_{e}}{d t}= & -n e(\mathbf{E}+\mathbf{V} \times \mathbf{B}-\eta \mathbf{J}) \\
& -\nabla p_{e}-\nabla \cdot \boldsymbol{\Pi}_{e}+\mathbf{J} \times \mathbf{B},
\end{aligned}
$$

to solve for $\mathbf{E}$ in a straightforward way. Substituting the resulting value for $\mathbf{E}$ into Faraday's equation results in a well-posed equation for the magnetic field $\mathbf{B}$. If we neglect electron inertia and introduce the auxiliary fields $\hat{\mathbf{E}}$ and $\hat{\mathbf{B}}$ defined by ${ }^{74}$

$$
\hat{\mathbf{E}}=-\mathbf{V} \times \mathbf{B}+\mathbf{J} \times \hat{\mathbf{B}} / n e-\left(\nabla p_{e}+\nabla \cdot \boldsymbol{\Pi}_{e}\right) / n e+\eta \mathbf{J}
$$

and

$$
\hat{\mathbf{B}}=\left(1-d_{e}^{2} \nabla^{2}\right) \mathbf{B},
$$

the result takes a form reminiscent of Faraday's equation:

$$
\frac{\partial \hat{\mathbf{B}}}{\partial t}=-\nabla \times \hat{\mathbf{E}} .
$$

In addition to its use in fluid models, the above result is also commonly used in hybrid kinetic simulations where the ions are treated with a kinetic formulation while the electrons are assumed to obey Eq. (27) with some fluid closure for $\Pi_{e}$ (Ref. 73).

To summarize, we see that the quasi-neutral and subluminal approximations describe two different physical circumstances and that they may, in principle, be used separately. For the low-frequency dynamics considered in this lecture, however, both approximations apply, and their joint use results in a simple and elegant formulation of the remaining field equation.

A similar reduction procedure may be followed to eliminate the compressional Alfvén wave, leading to the reduced MHD model. ${ }^{22,23}$ Comparison of the dispersion relation for the compressional Alfvén wave, $\omega=$ $k V_{A}$, to that for the shear Alfvén wave, $\omega=k_{\|} V_{A}$, shows that the separation of scales for these two waves requires that the flute ordering be satisfied, $k_{\|} \ll k$. As discussed in Sec. I, this ordering is generally regarded by the MHD community as unrealistic when applied 
outside the narrow boundary layers surrounding the resonant surfaces. We nevertheless adopt it for the gyrofluid model presented in Sec. V, in order to examine the nonlocal effects of ion gyration that are omitted from the fluid models discussed in the present section.

\section{III.C. The Drift Model}

The drift model is a special case of a family of closures called two-fluid models. Its aim is to describe disturbances such that the plasma velocity

$$
V \sim V_{D} \ll V_{A},
$$

where $V_{D}=|\mathbf{b} \times \nabla p| / e B n$ is the drift velocity. This distinguishes it from other two-fluid models for which the velocity is unrestricted. Before further describing the drift model, however, it is useful to place it in context by noting that all fluid models in a single-species plasma are "two-fluid" models except, in a sense, MHD. The latter assertion may seem surprising since, after all, MHD describes a conducting fluid and its equations are unmistakably different from the Navier-Stokes equations that also purport to describe a single fluid. In particular, the ion and electron velocity and density are distinct and can all be computed from the MHD fields using $n_{i}-n_{e}=$ $\epsilon \nabla \cdot \mathbf{E} / e$ and $\mathbf{V}_{i}-\mathbf{V}_{e}=\mathbf{J} / n e$. So in what sense is the MHD model "single fluid"? The answer is that the difference in the velocity of the ions and electrons is small compared to the plasma velocity, which in MHD is assumed to be of order $V_{t i}$, the ion thermal velocity. To be specific, we may estimate the relative difference as follows:

$$
\left(V_{i}-V_{e}\right) / V_{i} \sim J / n e V_{t i} \sim d_{i} / L \beta_{i}^{1 / 2}=\rho_{i} / L \beta_{i} \ll 1 .
$$

This estimate shows that the relative velocity difference in MHD is small, although not as small as the relative charge separation computed in Eq. (25). In conclusion, MHD should be thought of as a "quasi-single-fluid" model, in the same sense that it is a "quasi-neutral" model. Returning to the drift model, we note that its value and distinctiveness lies not in the multiplicity of fluids it describes but rather in the sophisticated way in which it uses magnetization to infer closure relations for many of the high-order moments.

The drift model ${ }^{21,75}$ is a rigorous closure of the moment equations based on the Chapman-Enskog procedure. Note that in gas-kinetic theory, the ChapmanEnskog procedure requires that the scale lengths be smaller than the mean-free path in every direction. The drift model relaxes this condition considerably by relying on the Larmor gyration to enforce isotropy in the directions perpendicular to the magnetic field. The conditions required for its validity are that the Larmor radius be small compared to the smallest-length scales in the plasma and that the mean-free path be much shorter than the scale of variation in the parallel direction:

$$
k_{\mathrm{\|}} \lambda_{\mathrm{mfp}} \ll 1
$$

and

$$
k_{\perp} \rho_{i} \ll 1
$$

Unfortunately, the first of these conditions is seldom applicable in fusion experiments, and as we have discussed, many modes of interest violate the second condition in the resonant layers. As a result, in the context of fusion theory, the drift model is generally regarded as a truncation of the moment hierarchy, and some of the terms involving higher moments are often discarded for simplicity. It does, however, offer a good description of the perpendicular dynamics, and this enables it to account for many observed phenomena.

The key idea behind the description of the perpendicular dynamics in the drift model is to use the dominance of the terms proportional to $\omega_{c s}$ in the moment equations to express some of the moments in terms of lower-order moments. ${ }^{76}$ Thus, the moment equations may be rearranged to solve for $\mathbf{M}_{s}^{(k)}$ :

$$
\llbracket \mathbf{b} \times \mathbf{M}_{s}^{(k)} \rrbracket=-\frac{1}{\omega_{c s}} \mathbf{S}_{s}^{(k)},
$$

where

$$
\mathbf{S}_{s}^{(k)}=\frac{\partial \mathbf{M}_{s}^{(k)}}{\partial t}+\nabla \cdot \mathbf{M}_{s}^{(k+1)}+\frac{e_{s}}{m_{s}} \llbracket \mathbf{E M}_{s}^{(k-1)} \rrbracket-\mathbf{C}^{(k)} .
$$

The value of this approach is that due to the factor of $1 / \omega_{c s} \ll 1$, the moment on the left side can be evaluated to any given accuracy in $\rho_{s} / L$ from the knowledge of the right side to one lesser degree of accuracy in $\rho_{s} / L$.

Unfortunately, the operator applied to the moment on the left side of Eq. (30) is not invertible, so that the homogeneous equation

$$
\llbracket \mathbf{b} \times \mathbf{M}_{s}^{(k)} \rrbracket=0
$$

has nontrivial solutions. The moments may thus be separated into two contributions: the solution of the homogeneous Eq. (32) and the inhomogeneous solution.

The calculations involved in the solution of the moment Eqs. (30) and (31) are laborious and have been described at length by other authors. Our purpose here is merely to give a flavor of the arguments involved in their derivation, particularly as regards the geometric origins of their complicated tensorial structure, in the hope of making their appearance less forbidding. To this end, we briefly sketch the derivation of the gyroviscosity tensor, omitting the species index for simplicity.

The gyroviscosity tensor is related to the secondorder moment by

$$
\boldsymbol{\Pi}_{g} \equiv \mathbf{P}-m n \mathbf{V V}+\frac{1}{2} m n V^{2} \mathbf{I}
$$


where $\mathbf{P}=\mathbf{M}^{(2)}$. We first note that the homogeneous solution of Eq. (32) for $k=2$ can be seen to be the gyrotropic tensor, which takes the form

$$
\mathbf{P}_{C G L}=p_{\perp}(\mathbf{I}-\mathbf{b b})+p_{\|} \mathbf{b b} .
$$

The inhomogeneous part of the solution, by contrast, takes the form

$$
\mathbf{P}_{\text {inhom }}=\frac{1}{\omega_{c}}\left[\mathbf{b} \times \mathbf{S}^{(2)}+3 \mathbf{b b} \times\left(\mathbf{b} \cdot \mathbf{S}^{(2)}\right)\right] .
$$

The pressure tensor can thus be calculated from the firstorder solution of the kinetic equation. Direct evaluation of the elements of $\mathbf{M}^{(3)}$ that appear in $\mathbf{S}^{(2)}$ shows that the only contributions to this term come from the lowestorder solution of the kinetic equation given by $\tilde{f}=$ $-\rho_{s} \cdot \nabla f_{M}$, where $f_{M}$ is the background Maxwellian distribution. Using Eq. (33) leads then to the following expression of the gyroviscosity:

$$
\boldsymbol{\Pi}_{g v}=\boldsymbol{\Pi}_{\perp}+\mathbf{b} \Pi_{\|}+\Pi_{\|} \mathbf{b} .
$$

Here $\Pi_{\perp}$ is the tensor

$$
\begin{aligned}
\Pi_{\perp}= & \frac{p}{4 \omega_{c}}\left[(\mathbf{b} \times \nabla) \mathbf{V}_{\perp}+\nabla\left(\mathbf{b} \times \mathbf{V}_{\perp}\right)+\text { transpose }\right] \\
& +\frac{1}{10 \omega_{c}}\left[(\mathbf{b} \times \nabla) \mathbf{q}_{\perp}+\nabla\left(\mathbf{b} \times \mathbf{q}_{\perp}\right)+\text { transpose }\right],
\end{aligned}
$$

where $\mathbf{q}_{\perp}$ is the perpendicular component of the heat flux,

$$
\mathbf{q}_{\perp}=-\frac{5}{2} \frac{p}{e B} \mathbf{b} \times \nabla T
$$

and $\Pi_{\|}$is the vector

$$
\begin{aligned}
\Pi_{\|}= & \frac{p}{\omega_{c}}\left[\mathbf{b} \times \nabla V_{\|}+\mathbf{b} \cdot \nabla\left(\mathbf{b} \times \mathbf{V}_{\perp}\right)\right] \\
& +\frac{1}{5 \omega_{c}} \mathbf{b} \cdot \nabla\left(\mathbf{b} \times \mathbf{q}_{\perp}\right) .
\end{aligned}
$$

We note that the form of the gyroviscosity given by Braginskii is based on the MHD ordering, where the electric drift (i.e., the $\mathbf{E} \times \mathbf{B}$ velocity) is assumed to dominate. ${ }^{77}$ The present version, appropriate to the drift ordering, was first derived by Mikhailovskii. ${ }^{78}$ Improved versions of the drift model have been derived recently by several authors. ${ }^{79-82}$ We also note that Pogutse and collaborators $^{83}$ have shown that retaining the (formally small) inertial terms in the pressure tensor leads to a nonlocal description of the effects of finite Larmor radius that is similar in form to the gyrofluid equations that we will present in Sec. V. Similarly to the gyrofluid model and unlike Mikhailovskii's equations, the model of Pogutse et al. leads to dispersion relations that offer a good approximation to their kinetic counterpart. ${ }^{83}$

In Sec. V, we present an alternative approach to the development of fluid models, based on taking the moments of the gyrokinetic equation. In the following (Sec. IV), we begin by giving a description of the gyrokinetic model.

\section{THE GYROKINETIC MODEL}

The gyrokinetic equation is a reduction of the MaxwellVlasov system of equations obtained by averaging these equations over the rapid time scale corresponding to Larmor gyration. ${ }^{84-86}$ The averaging procedure allows for perturbations with small wavelengths across the magnetic field, $k_{\perp} \rho_{s} \sim 1$, provided such perturbations have small amplitude (otherwise, the particles would cease exhibiting identifiable Larmor gyrations; i.e., they would be demagnetized). Because of the presence of the shortwavelength perturbation, the guiding-center variables acquire a dependence on the rapidly varying gyration phase. In order to preserve the phase-space conservation properties of the Vlasov equation, the reduction procedure transforms the equations to a new set of slowly varying variables, called the gyro-center variables. In the limit where only long wavelengths are present, $k_{\perp} \rho_{s} \ll 1$, the gyrokinetic model reduces to the drift kinetic model that serves as the foundation for the theory of neoclassical transport. One might expect that the restriction on the amplitude of the perturbations would lead to a system that is effectively linear, but the singularity of the leading-order equations near closed field lines results in the nonlinear terms being important there. Thus, although the gyrokinetic equation is independent of the flute ordering, the interesting nonlinear dynamics it describes occur in regions where this ordering is satisfied.

Formulated in terms of the canonical momentum $\mathcal{P}_{\|}$, the gyrokinetic equation takes the form

$$
\frac{d f}{d t} \equiv \frac{\partial f}{\partial t}+\frac{d \mathbf{R}}{d t} \cdot \nabla f+\frac{d \mathcal{P}_{\|}}{d t} \frac{\partial f}{\partial \mathcal{P}_{\|}}=0,
$$

where $f$ represents the distribution of gyro-centers. The evolution of the gyro-centers is governed by

$$
\frac{d \mathbf{R}}{d t}=v_{\|} \frac{\mathbf{B}^{\star}}{B_{\|}^{\star}}+\frac{\mathbf{b}}{e B_{\|}^{\star}} \times\left(\mu \nabla B+e \nabla\left\langle\Psi_{g y}\right\rangle_{s}\right)
$$

and

$$
\frac{d \mathcal{P}_{\|}}{d t}=-\frac{\mathbf{B}^{\star}}{B_{\|}^{\star}} \cdot\left(\mu \nabla B+e \nabla\left\langle\Psi_{g y}\right\rangle_{s}\right) .
$$

Here $\mu=m v_{\perp}^{2} / 2 B, \mathbf{B}^{\star}=\nabla \times \mathbf{A}^{\star}$, and $B_{\|}^{\star}=\mathbf{b} \cdot \mathbf{B}^{\star}$, where $\mathbf{A}^{\star}=\mathbf{A}+\left(\mathcal{P}_{\|} / e\right) \mathbf{b}$ and

$$
v_{\|}=\frac{1}{m}\left(\mathcal{P}_{\|}-e\left\langle A_{\|}\right\rangle\right) .
$$


The effective perturbation potential $\Psi_{g y}$ is

$$
\left\langle\Psi_{g y}\right\rangle_{s} \equiv\langle\phi\rangle_{s}-\mathcal{P}_{\|}\left\langle A_{\|}\right\rangle_{s} / m
$$

The gyro-averaged potentials are defined by

$$
\langle\phi\rangle_{s}(\mathbf{R}, \mu) \equiv \frac{1}{2 \pi} \oint \phi\left(\mathbf{R}+\boldsymbol{\rho}_{s}\right) d \alpha
$$

and

$$
\left\langle A_{\|}\right\rangle_{s}(\mathbf{R}, \mu) \equiv \frac{1}{2 \pi} \oint A_{\|}\left(\mathbf{R}+\boldsymbol{\rho}_{s}\right) d \alpha .
$$

Lastly, the fields are determined by the quasi-neutrality constraint and by Ampère's law,

$$
\sum_{s}\left[n_{s}+\frac{e_{s} n_{0}}{T_{s}}\left(\langle\bar{\phi}\rangle_{s}-\phi\right)\right]=0
$$

and

$$
\sum_{s}\left(\frac{\beta_{s}}{\rho_{s}^{2}}\left\langle\bar{A}_{\|}\right\rangle_{s}-\mu_{0}\left\langle J_{\| s}\right\rangle_{s}\right)=\nabla^{2} A_{\|},
$$

where the notation $\langle\bar{\xi}\rangle_{s}$ denotes the average of the field $\xi$ over the Larmor orbits and the background Maxwellian of species $s$.

The gyrokinetic model is widely used in the simulation of turbulence in magnetic fusion devices, ${ }^{87}$ and it is beginning to be used in the study of turbulence in the solar wind and other astrophysical applications. ${ }^{88}$ The numerical methods used to solve the gyrokinetic equations divide into PIC methods, the Eulerian method, ${ }^{89,90}$ and the semi-Lagrangian method ${ }^{87}$ The PIC methods are named after the computational macroparticles that they use to advance the distribution function. It is important to note, however, that unlike the so-called molecular dynamics codes that account for all interparticle forces, the computational particles evolve under the action of the mean fields only. That is, despite its misleading name, the PIC method solves a continuum equation, the gyrokinetic equation, that describes the evolution of a differentiable density $f$ in phase space. The use of computational particles can be interpreted as an application of the Monte Carlo method to the calculation of the integrals that form the moments of the distribution function..$^{91}$ This interpretation sheds light on the methods used to reduce noise and informs ongoing research on algorithmic improvements. ${ }^{92}$ We briefly describe the Monte Carlo method in order to expose the differences between the Eulerian and PIC methods.

The Monte Carlo (MC) method for calculating integrals consists in summing the values of the integrand sampled at random points. The relative merits of this method are illustrated by the example of calculating the volume of a hypersphere of unit diameter using a strategy inspired by the "battleship" game. The strategy consists in scattering shots randomly across a hypercube with sides of length unity that encloses the hypersphere whose volume we seek to evaluate (see Fig. 10). The score is incremented by unity for every "strike," or every time a shot falls within the hypersphere [red dots in Fig. 10 (color online)]. At the end of the game, the volume of the sphere is estimated as $V=S / N$, the ratio of the score $S$ and the number of shots $N$. The standard deviation $\sigma$ for a single shot is clearly of order unity. The variance of the score is $\left\langle(S-\langle S\rangle)^{2}\right\rangle=N \sigma^{2}$, where $\langle S\rangle$ is the average of the volumes calculated over a large number of games. The standard deviation in the estimate for the volume thus has the scaling

$$
(\delta V)_{\mathrm{MC}} \sim \sigma N^{-1 / 2} .
$$

We next compare the accuracy of the MC approximation to that of a deterministic method based on a firstorder accurate integration algorithm, such as those used in shock-capturing methods. The deterministic method divides the enclosing volume into a grid of $N$ cells, each of size $h$, where $N=h^{-d}$. It subsequently estimates the volume of the hypersphere as the product of the volume of the cells and the number of cells that lie within the hypersphere. The error is then proportional to the product of the volume of the cells, $h^{d}$, and the number of cells lying across the surface of the sphere, $(1 / h)^{d-1}$, since

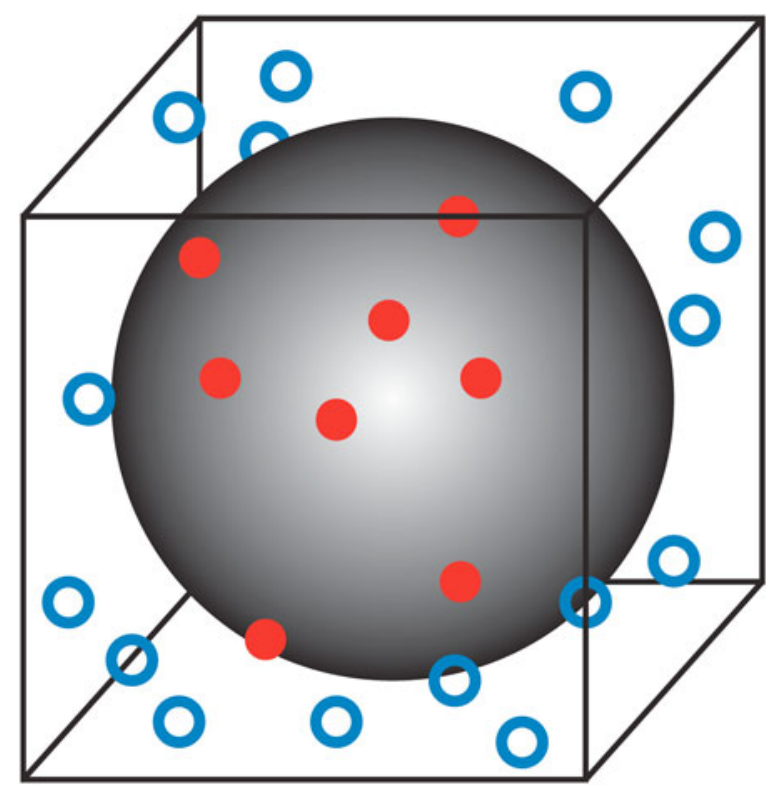

Fig. 10. Illustration of an application of the Monte Carlo method to calculate the volume of a hypersphere for dimension $d=3$. The closed circles represent hits, or points falling within the sphere, and the open circles represent misses, or points falling outside the sphere. The ratio of the number of hits to the total number of points approaches $\pi / 6$ when the number of points $N \rightarrow \infty$. 
part of the volume of these bounding cells should be included in the total. It follows that the error scales like $h$, or

$$
(\delta V)_{\text {grid }} \sim N^{-1 / d} .
$$

In conclusion, we see that the MC method is competitive with a first-order accurate Eulerian method for $d=2$, precisely the dimensionality of the $\left(v_{\perp}, v_{\|}\right)$integrals called for by the gyrokinetic method. In the case of the full Vlasov equation, by contrast, $d=3$ and the PIC method has an advantage. This advantage may be offset, however, by using a higher-order algorithm in the Eulerian method.

Following early work by Naitou et al. ${ }^{93}$ on the internal kink and by Sydora on microscopic magnetic islands, ${ }^{94}$ several efforts have been undertaken to apply the gyrokinetic model more broadly to long-wavelength modes. ${ }^{95}$ One of the challenges involves the treatment of the very rapid streaming of the electrons along the magnetic field. ${ }^{96-99}$ Recent advances have enabled the simulation of Alfvén eigenmodes. ${ }^{92}$ More widespread investigations of the gyrokinetic dynamics of longwavelength disturbances appear likely to lead to important insights in the years preceding ITER operations.

\section{THE GYROFLUID MODEL}

It has been known for some time that taking the moments of the gyrokinetic equation yields reduced models for the plasma dynamics, the so-called gyrofluid models, that include the nonlocal response of the plasma resulting from particle gyration. ${ }^{100-102}$ After their discovery, these models enjoyed a brief period of success in the context of simulations of turbulent transport. They were abandoned, however, when it was discovered that they overestimated the damping of the zonal flows, thereby overestimating the overall strength of the turbulent transport. ${ }^{32,33,103}$ More recently, gyrofluid models have enjoyed a resurgence in applications where nonlinear electromagnetic effects are important, ${ }^{104,105}$ such as in the study of magnetic reconnection. ${ }^{61,106,107}$ In this section we describe a simple version of this model obtained by keeping only a few low-order moments.

A distinctive feature of gyrofluid closures is that due to the nonalgebraic dependence of the gyro-averaging operator on the velocity, the moment equations at every order, including the lowest, contain moments of arbitrarily high order. This is in contrast to the conventional moment hierarchy, where each equation for the $n$ 'th moment contains terms involving at most the $n+1$ 'th moment. For example, in gyrofluid theories the lowestorder moment equation takes the form

$$
\frac{d n}{d t}+\overline{\mathbf{b}} \cdot \nabla u_{\|}+\frac{1}{2}\left(\hat{\nabla}_{\perp}^{2} \overline{\mathbf{v}}\right) \cdot \nabla T_{\perp}+\ldots=0
$$

where

$$
\frac{d}{d t}=\frac{\partial}{\partial t}+\overline{\mathbf{v}} \cdot \nabla
$$

is the convective derivative along the orbit-averaged velocity $\overline{\mathbf{v}}$, itself expressed in terms of the orbit-averaged electrostatic potential $\Phi=\Gamma_{0}^{1 / 2} \phi$ according to

$$
\overline{\mathbf{v}}=\mathbf{b}_{0} \times \nabla \Phi
$$

The magnetic field is

$$
\overline{\mathbf{b}}=\left(\mathbf{B}_{0}+\mathbf{B}_{0} \times \nabla \Psi\right) / B_{0},
$$

where $\Psi=\Gamma_{0}^{1 / 2} \psi$ is the orbit-averaged projection of the magnetic potential along the magnetic field.

Dorland and Hammett showed that the gyro-averaging operations could be modeled by the operator $\Gamma_{0}^{1 / 2}$ defined as follows ${ }^{101}$ :

$$
\Gamma_{0}^{1 / 2} \xi=\exp \left(\frac{1}{2} \tau \nabla_{\perp}^{2}\right) I_{0}^{1 / 2}\left(-\tau \nabla_{\perp}^{2}\right) \xi
$$

where $\tau=T_{s} / T_{\text {ref }}$ and $I_{0}$ is the modified Bessel function of the first kind. The definition in Eq. (45) may be interpreted in terms of its series expansion,

$$
\Gamma_{0}^{1 / 2}=1+\sum_{n=1}^{\infty} a_{n}\left(\tau \nabla_{\perp}^{2}\right)^{n}=1+(\tau / 2) \nabla^{2}+\cdots .
$$

In practice, the definition Eq. (45) is most easily implemented in spectral or pseudo-spectral codes where $\Gamma_{0}^{1 / 2}(k)$ acts on the fields as a simple multiplier that can be evaluated directly in terms of the norm $k$ of the wavevector. In finite difference or finite element codes, by contrast, the action of $\Gamma_{0}^{1 / 2}$ must be evaluated using an approximate form, usually the lowest-order Padé approximant:

$$
\Gamma_{0}^{1 / 2}=\left(1+\frac{1}{2} \rho_{i}^{2} \nabla^{2}\right)^{-1}
$$

This operator is straightforward to evaluate numerically.

\section{V.A. The Electromagnetic Gyrofluid Model}

We present a simple gyrofluid model ${ }^{108}$ that illustrates the physics described by such models and, we hope, will motivate the development of more detailed and realistic models. In order to keep the presentation simple, we omit the Landau damping and collisional transport terms from the presentation; for the most part, restoring these terms is straightforward, if not trivial. Keeping only the first two moments of the gyrokinetic equations for electrons and ions and neglecting the electron gyroradius and field-line curvature yields the following simple and physically transparent model: 


$$
\begin{array}{r}
\frac{\partial n_{i}}{\partial t}+\overline{\mathbf{v}}_{E} \cdot \nabla n_{i}=0, \\
\frac{\partial n_{e}}{\partial t}+\mathbf{v}_{E} \cdot \nabla n_{e}-V_{A}^{2} \nabla_{\|} J=0,
\end{array}
$$

and

$$
\frac{\partial}{\partial t}\left(\psi-d_{e}^{2} J\right)-d_{e}^{2} \mathbf{v}_{E} \cdot \nabla J+\nabla_{\|}\left(n_{e}-\phi\right)=0,
$$

where

$$
\begin{aligned}
n_{i} & =\text { density of ion guiding centers } \\
n_{e} & =\text { electron particle density } \\
\psi & =\text { parallel component of the vector potential. }
\end{aligned}
$$

The system is closed by Ampère's law,

$$
J=\nabla^{2} \psi,
$$

and the quasi-neutrality equation,

$$
n_{e}=\Gamma_{0}^{1 / 2} n_{i}+\left(\Gamma_{0}-1\right) \phi / \tau .
$$

The two terms on the right side of Eq. (49) both represent contributions to the ion particle density, which differs from the gyro-center density $n_{i}$ due to the gradient of the local electric field that the ions experience along their orbit. The resulting acceleration of the ions along their orbits leads to a difference between the gyro-center and particle densities, called the polarization density, which is represented by the last term in Eq. (49).

The physical interpretation of the various equations is as follows. The first, Eq. (46), is easily recognized as the ion continuity equation, in which the ion density is convected by the velocity composed of the electric drift velocity. Naturally, due to their Larmor gyration, the ions advance under the effect of the orbit-averaged value of the electric drift. Note that the contribution of the parallel velocity of the ions to the divergence of the particle flux is neglected in Eq. (46). This has the effect of eliminating sound waves from the model and is justified when the sound wave frequency is much smaller than the frequency of the perturbations, $k_{\|} c_{s} \ll \omega$. When this condition is satisfied, the inertia of the ions inhibits them from flowing along the field lines in response to pressure imbalances, and their velocity along the magnetic field remains small. This condition is often satisfied in the singular layers and in thin magnetic islands.

The second, Eq. (47), is the electron continuity equation expressing the conservation of electron mass. Since the present model neglects the electron gyroradius, the electrons are convected by the local value of the electric drift and by the parallel flow, which is proportional to the current since the model neglects the ion velocity. Note that the electric drift is solenoidal $\left(\nabla \cdot \mathbf{v}_{E}=0\right)$, so that the compression of the electron fluid is due entirely to the last term, representing the effect of the flow of electrons along the magnetic field in response to the parallel forces in the momentum equation.

The third and last, Eq. (48), describes the conservation of electron momentum and is often referred to as Ohm's law. Recalling the relationship of $J$ to the electron velocity, we see that the combination $\psi-d_{e}^{2} J$ is proportional to the canonical momentum density of the electrons. The first and last terms in Eq. (48) can be recognized as the inductive and electrostatic parts of the parallel component of the electric field, respectively. The two terms involving the currents form the total derivative of the electron momentum along the trajectory of the electron fluid. Lastly, the parallel gradient of the density represents the pressure forces acting on a fluid element.

It is instructive to examine the cold-ion limit of the model. In this limit, the polarization density reduces to

$$
\lim _{\tau \rightarrow 0}\left(\Gamma_{0}-1\right) \phi / \tau=\nabla^{2} \phi
$$

and $\Gamma_{0}^{1 / 2} \rightarrow 1$. The quasi-neutrality Eq. (49) thus takes the form

$$
n_{e}-n_{i}=\nabla^{2} \phi
$$

The fact that we recover Poisson's equation despite having assumed quasi-neutrality is explained by the distinction between the "free" charge represented by the gyro-centers and the actual space charge. The difference between the free and the total charge density is due to the polarizability of the guiding center plasma and may be thought of as a "bound" charge associated with the gyro-center.

Taking the time derivative of the quasi-neutrality equation and eliminating $n_{e}$ and $n_{i}$ with the continuity equations yields the vorticity equation,

$$
\frac{\partial \Omega}{\partial t}+\mathbf{v}_{E} \cdot \nabla \Omega=V_{A}^{2} \nabla_{\|} J
$$

also referred to as the shear Alfvén equation. Here,

$$
\Omega=\hat{\mathbf{z}} \cdot \nabla \times \mathbf{v}_{E}=\nabla^{2} \phi
$$

is the component of the vorticity of the plasma along the background magnetic field. The meaning of this equation can be understood by thinking of the parallel component of the current $J$ as a measure of the winding density of neighboring field lines. That is, if we adopt a particular field line as our reference, the neighboring field lines will wind around it like springs with a winding density measured by $J$. If the winding density is tighter down the magnetic field than it is up the magnetic field, the gradient of the tension in the wound field lines will act to spin up the plasma in between, thereby increasing the vorticity $\Omega$ and relaxing the inhomogeneity in the tension. 


\section{V.B. Role of the Hamiltonian Property}

A fundamental property of plasma, viewed as a dynamical system of charged particles, is that it is governed by Hamilton's equations. That is, the equations of motion may be written in the form

$$
\dot{X}_{j}=\left\{H, X_{j}\right\},
$$

where $H(\mathbf{X})$ is the Hamiltonian, the $X_{j}$ are the dynamical variables, and $\{\cdot, \cdot\}$ represents the Poisson brackets. This has important physical consequences, such as the existence of Poincaré invariants of the motion. In the collisionless limit, the Hamiltonian property is inherited by the Maxwell-Vlasov model. ${ }^{109}$ In the ideal and inviscid limit, it is likewise inherited by the MHD model.

In view of the above considerations, demanding that any prospective fluid model satisfy the Hamiltonian property in the limit of vanishing collision frequency appears to be a natural constraint on the closure scheme. ${ }^{10}$ Clearly, this demand is also highly selective. ${ }^{26}$ Furthermore, the Hamiltonian property confers some very desirable qualities to the model. The most important of these is that it guarantees the solvability of the equilibrium problem. Since we have seen that long-wavelength plasma dynamics often proceeds through a sequence of equilibrium or near-equilibrium states, ensuring the solvability of the equilibrium problem is clearly a high priority for any prospective model, equal in importance to the conservation of energy and momentum.

In order to provide the context in which to present the link between the Hamiltonian property and the equilibrium solutions, we begin by describing the solvability problem for the equilibrium problem. In a sense, we already confronted this problem in Sec. I when we explained the origin of the magnetic resonances near closed field lines. The point is that although the force balance condition is satisfied by the perpendicular current calculated in Eq. (1), it is by no means obvious that a finite solution exists for the parallel current needed to satisfy the continuity equation, $\nabla \cdot \mathbf{J}=0$. That is, the problem of the solubility of the MHD equilibrium equations reduces to the question of the existence of a solution to the magnetic differential Eq. (2). Clearly, in order for this equation to possess a single-valued solution, it is necessary that the integral of the right side along any closed field line vanish:

$$
\oint \frac{d \ell}{B} \nabla \cdot J_{\perp}=0
$$

It is easy to see that similar conditions occur for the gyrofluid model presented here. For example, the electron continuity requires that the integral of $\mathbf{v}_{E} \cdot \nabla n_{e}$ vanish along any closed field line, and Ohm's law requires the same of $\mathbf{v}_{E} \cdot \nabla J$. Similar solubility conditions can also be formulated in terms of integrals along closed stream lines. Verifying such solubility conditions is sometimes possible. Alternatively, it is occasionally possible to demonstrate that a given model violates the equilibrium solubility conditions. ${ }^{111}$ More commonly, however, the question cannot be resolved.

A virtue of the Hamiltonian property is that it not only guarantees the solvability of the equilibrium equations but also provides first integrals of these equations. In the case of MHD, for example, the Hamiltonian formulation automatically guarantees that the solubility condition of Eq. (50) is satisfied and provides the integral of Eq. (2), which is none other than the Grad-Shafranov equation. ${ }^{12}$ To obtain these "first integrals" of the equilibrium equations, we note that any extremum of the Hamiltonian is automatically an equilibrium solution, since the Poisson bracket acts on the functional derivative of its arguments. Thus, the Poisson bracket vanishes automatically when acting on an extremal functional. In order to obtain the most general solution possible, however, it is necessary to add to the Hamiltonian a linear combination of a family of functionals $C(\mathbf{X})$, called Casimirs, that are defined by the property that

$$
\left\{X_{j}, C(\mathbf{X})\right\}=0
$$

for all $j$, or, equivalently, that their Poisson bracket with any other functional vanishes. These functionals describe quantities that are geometric invariants of the system. That is, they are conserved regardless of the value of the Hamiltonian, since

$$
\frac{d C}{d t}=\frac{d \mathbf{X}}{d t} \cdot \nabla C=\{C, H\} \equiv 0 .
$$

Physically, Casimirs represent conserved quantities such as the magnetic flux or the particle mass. Examples of the use of the Hamiltonian property to solve the equilibrium equations can be found in Refs. 111 and 113 .

In conclusion, we see that the Hamiltonian formulation of a dynamical system leads to a new formulation of the equilibrium condition,

$$
\frac{\delta F}{\delta \mathbf{X}}=0
$$

where

$$
F(\mathbf{X})=H(\mathbf{X})+C(\mathbf{X}) .
$$

The variational form of the equilibrium equation does not suffer from the problem with solubility conditions and yields directly the first integrals of the equations obtained by setting the time derivative to zero in the dynamical equations. Furthermore, the second variation of $F(\mathbf{X})$ provides information on stability. ${ }^{114}$ Since $F$ is conserved, the positive definiteness of the second variation,

$$
\delta^{2} F>0
$$


guarantees stability. In the case of MHD, the second variation of $F$, when expressed in terms of the displacement field $\boldsymbol{\xi}$, yields the well-known energy principle.

\section{SUMMARY}

The predictive modeling of sub-Alfvénic plasma motions presents a formidable challenge due to the large number of scales involved and the need to account for kinetic effects as well as for the effects of coupling to turbulent fluctuations. The techniques available for dealing with the multiple scales can be broadly divided into analytic reduction methods and numerical overstriding. The most successful codes combine both techniques, using reduction to eliminate space-charge oscillations, light waves, and cyclotron oscillations, and using implicit or semi-implicit algorithms to overcome the limitations placed by the Alfvén and whistler waves. Kinetic methods, only briefly mentioned in this overview, are being developed but have not reached maturity for longwavelength dynamics. Gyrofluid models, originally developed for the study of turbulence, appear to present attractive possibilities for extending the capabilities of two-fluid codes without incurring the cost associated with the kinetic approach. In order to be applicable in regions where the flute ordering is violated, however, these models need to be extended to include the quasi-static effects associated with the compressional Alfvén wave.

\section{ACKNOWLEDGMENTS}

This lecture benefited from discussions with many colleagues, including R. Fitzpatrick, E. Lazarus, and C. Sovinec. The work was supported by the U.S. Department of Energy under grant DE-FG02-04ER-54742.

\section{REFERENCES}

1. T. C. HENDER et al., Nucl. Fusion, 47, S128 (2007).

2. J. FREIDBERG, Rev. Mod. Phys., 54, 801 (1982).

3. M. J. LANCTOT et al., Phys. Plasmas, 17, 030701 (2010).

4. A. D. TURNBULL et al., Nucl. Fusion, 42, 917 (2002).

5. P. B. SNYDER et al., Phys. Plasmas, 3, 2037 (2002).

6. G. T. A. HUYSMANS, J. P. GOEDBLOED, and W. KERNER, Phys. Fluids B: Plasma Phys., 5, 1545 (1993).

7. W. KERNER et al., J. Comput. Phys., 142, 271 (1998).

8. D. H. LIU and A. BONDESON, Comput. Phys. Commun., 116, 55 (1999).

9. A. Y. AYDEMIR, Phys. Rev. Lett., 59, 649 (1987).

10. K. LERBINGER AND J. LUCIANI, J. Comput. Phys., 97, 444 (1991).

11. W. PARK et al., Phys. Plasmas, 6, 1796 (1999).

12. A. H. GLASSER et al., Plasma Phys. Control. Fusion, 41, A747 (1999).
13. H. LÜTJENS and J.-F. LUCIANI, J. Comput. Phys., 227, 6944 (2008).

14. W. PARK et al., Nucl. Fusion, 43, 483 (2003).

15. C. R. SOVINEC et al., Phys. Rev. Lett., 94, 035003 (2005).

16. E. B. HOOPER et al., Phys. Plasmas, 15, 032502 (2008).

17. J. A. BRESLAU, S. C. JARDIN, and W. PARK, Phys. Plasmas, 14, 056105 (2007).

18. O. SAUTER et al., Phys. Plasmas, 4, 1654 (1997).

19. D. P. BRENNAN, S. E. KRUGER, T. A. GIANAKON, and D. D. SCHNACK, Nucl. Fusion, 45, 1178 (2005).

20. J. F. DRAKE and R. G. KLEVA, Phys. Rev. Lett., 66, 1458 (1991).

21. R. D. HAZELTINE and J. D. MEISS, Phys. Reports, 121, 1 (1985).

22. H. R. STRAUSS, Phys. Fluids, 19, 134 (1976).

23. H. R. STRAUSS, Phys. Fluids, 20, 1354 (1977).

24. J. F. DRAKE and T. M. ANTONSEN, Phys. Fluids, 27, 898 (1984). 25. R. D. HAZELTINE, M. KOTSCHENREUTHER, and P. J. MORRISON, Phys. Fluids, 28, 2466 (1985).

26. R. D. HAZELTINE, C. T. HSU, and P. J. MORRISON, Phys. Fluids, 30, 3204 (1987).

27. P. ZHU et al., Phys. Rev. Lett., 101, 085005 (2008).

28. A. ISHIZAWA and N. NAKAJIMA, Phys. Plasmas, 14, 040702 (2007).

29. F. L. WAELBROECK, F. MILITELLO, R. FITZPATRICK, and W. HORTON, Plasma Phys. Control. Fusion, 51, 015015 (2009).

30. M. MURAGLIA et al., Phys. Rev. Lett., 103, 145001 (2009).

31. G. W. HAMMETT, W. DORLAND, and F. W. PERKINS, Phys. Fluids B, 4, 2052 (1992).

32. M. N. ROSENBLUTH and F. L. HINTON, Phys. Rev. Lett., 80, 724 (1998)

33. A. M. DIMITS et al., Phys. Plasmas, 7, 969 (2000).

34. R. J. HASTIE et al., Phys. Fluids, 30, 1756 (1987).

35. J. D. CALLEN et al., Phys. Plasmas, 6, 2963 (1999).

36. W. PARK and S. C. JARDIN, Phys. Fluids, 26, 1871 (1983).

37. F. L. WAELBROECK, Phys. Fluids B: Plasma Phys., 1, 499 (1989).

38. S. CAPPELLO and D. F. ESCANDE, Phys. Rev. Lett., 85, 3838 (2000).

39. P. MARTIN et al., Nucl. Fusion, 43, 1855 (2003).

40. I. T. CHAPMAN et al., Nucl. Fusion, 50, 045007 (2010).

41. K. H. BURRELL et al., Phys. Plasmas, 12, 056121 (2005).

42. E. A. LAZARUS et al., Plasma Phys. Control. Fusion, 48, L65 (2006).

43. C. MERCIER, Nucl. Fusion, 1, 47 (1960).

44. F. L. WAELBROECK, Nucl. Fusion, 49, 104025 (2009).

45. Q. YU, Phys. Plasmas, 13, 062310 (2006).

46. L. N. TREFETHEN, A. E. TREFETHEN, S. C. REDDY, and T. A. DRISCOLL, Science, 261, 578 (1993).

47. F. L. WAELBROECK, J. Q. DONG, W. HORTON, and P. N. YUSHMANOV, Phys. Plasmas, 1, 3742 (1994).

48. S. J. CAMARGO, M. K. TIPPETT, and I. L. CALDAS, Phys. Plasmas, 7, 2849 (2000).

49. S. J. CAMARGO, M. K. TIPPETT, and I. L. CALDAS, Phys. Rev. E, 58, 3693 (1998).

50. E. CAMPOREALE, D. BURGESS, and T. PASSOT, Phys. Plasmas, 16, 030703 (2009). 
51. D. BORBA et al., Phys. Plasmas, 1, 3151 (1994).

52. O. S. JONES, U. SHUMLAK, and D. S. EBERHARDT, J. Comput. Phys., 130, 231 (1997).

53. L. CHACON, D. A. KNOLL, and J. M. FINN, J. Comput. Phys., 178, 15 (2002).

54. L. CHACÓN and D. A. KNOLL, J. Comput. Phys., 188, 573 (2003).

55. S. C. JARDIN and J. A. BRESLAU, Phys. Plasmas, 12, 056101 (2005).

56. S. C. JARDIN, J. BRESLAU, and N. FERRARO, J. Comput. Phys., 226, 2146 (2007).

57. G. T. A. HUYSMANS, S. PAMELA, E. VAN DER PLAS, and P. RAMET, Plasma Phys. Control. Fusion, 51, 124012 (2009).

58. D. S. HARNED and D. D. SCHNACK, J. Comput. Phys., 65, 57 (1986).

59. D. S. HARNED and Z. MIKIĆ, J. Comput. Phys., 83, 1 (1989).

60. D. D. SCHNACK et al., J. Comput. Phys., 70, 330 (1987).

61. N. F. LOUREIRO and G. W. HAMMETT, J. Comput. Phys., 227, 4518 (2008).

62. E. VANDEN-EIJNDEN, Commun. Math. Sci., 5, 495 (2007).

63. M. A. SHAY, J. F. DRAKE, and B. DORLAND, J. Comput. Phys., 226, 571 (2007).

64. E. A. LAZARUS, J. B. LISTER, and G. H. NEILSON, Nucl. Fusion, 30, 111 (1990).

65. E. A. LAZARUS et al., Phys. Fluids B: Plasma Phys., 3, 2220 (1991).

66. M. DENNY, Eur. J. Phys., 23, 339 (2002).

67. D. S. HARNED and W. KERNER, J. Comput. Phys., 60, 62 (1985).

68. E. J. CARAMANA, J. Comput. Phys., 96, 484 (1991).

69. C. R. SOVINEC et al., J. Comput. Phys., 195, 355 (2004).

70. P. MAGET et al., Phys. Plasmas, 14, 052509 (2007).

71. C. R. SOVINEC et al., Phys. Plasmas, 10, 1727 (2003).

72. G. LAPENTA, J. U. BRACKBILL, and P. RICCI, Phys. Plasmas, 13, 055904 (2006).

73. D. C. BARNES, J. CHENG, and S. E. PARKER, Phys. Plasmas, 15, 055702 (2008).

74. M. SHAY, J. DRAKE, B. ROGERS, and R. DENTON, J. Geophys. Res.-Space Phys., 106, 3759 (2001).

75. D. D. SCHNACK et al., Phys. Plasmas, 13, 058103 (2006).

76. R. D. HAZELTINE and J. D. MEISS, Plasma Confinement, Volume 86 of Frontiers in Physics, Addison Wesley, Reading, Massachusetts (1992).

77. S. I. BRAGINSKII, Reviews of Plasma Physics, Vol. 1, p. 285, Consultants Bureau, New York (1985).

78. A. B. MIKHAILOVSKII, Sov. Phys. JETP, 25, 623 (1967).

79. A. N. SIMAKOV and P. J. CATTO, Phys. Plasmas, 10, 4744 (2003).

80. P. J. CATTO and A. N. SIMAKOV, Phys. Plasmas, 11, 90 (2004).

81. J. J. RAMOS, Phys. Plasmas, 12, 112301 (2005).

82. J. J. RAMOS, Phys. Plasmas, 12, 052102 (2005).

83. I. POGUTSE, A. SMOLYAKOV, and A. HIROSE, J. Plasma Phys., 60, 133 (1998).
84. H. QIN, W. M. TANG, and G. REWOLDT, Phys. Plasmas, 5, 1035 (1998).

85. H. QIN, W. M. TANG, and G. REWOLDT, Phys. Plasmas, 6, 2544 (1999).

86. A. J. BRIZARD and T. S. HAHM, Rev. Mod. Phys., 79, 421 (2007).

87. X. GARBET, Y. IDOMURA, L. VILLARD, and T. WATANABE, Nucl. Fusion, 50, 043002 (2010).

88. T. TATSUNO et al., Phys. Rev. Lett., 103, 015003 (2009).

89. M. KOTSCHENREUTHER, G. REWOLDT, and W. M. TANG, Comput. Phys. Commun., 88, 128 (1995).

90. J. CANDY and R. E. WALTZ, J. Comput. Phys., 186, 545 (2003). 91. A. Y. AYDEMIR, Phys. Plasmas, 1, 822 (1994).

92. R. HATZKY, A. KONIES, and A. MISHCHENKO, J. Comput. Phys., 225, 568 (2007).

93. H. NAITOU, K. TSUDA, W. W. LEE, and R. D. SYDORA, Phys. Plasmas, 2, 4257 (1995).

94. R. D. SYDORA, Phys. Plasmas, 8, 1929 (2001).

95. W. W. LEE and R. A. KOLESNIKOV, Phys. Plasmas, 16, 044506 (2009).

96. I. MANUILSKIY and W. W. LEE, Phys. Plasmas, 7, 1381 (2000). 97. W. W. LEE, J. L. V. LEWANDOWSKI, T. S. HAHM, and Z. LIN, Phys. Plasmas, 8, 4435 (2001).

98. Y. CHEN and S. PARKER, Phys. Plasmas, 8, 2095 (2001).

99. W. W. LEE and H. QIN, Phys. Plasmas, 10, 3196 (2003).

100. T. S. HAHM, W. W. LEE, and A. BRIZARD, Phys. Fluids, 31, 1940 (1988).

101. W. DORLAND and G. W. HAMMETT, Phys. Fluids, 5, 812 (1993).

102. P. B. SNYDER and G. W. HAMMETT, Phys. Plasmas, 8, 3199 (2001).

103. A. M. DIMITS et al., Nucl. Fusion, 40, 661 (2000).

104. B. D. SCOTT, Phys. Plasmas, 12, 102307 (2005).

105. T. PASSOT and P. L. SULEM, Phys. Plasmas, 14, 082502 (2007). 106. D. GRASSO, F. CALIFANO, F. PEGORARO, and F. PORCELLI, Plasma Phys. Reports, 26, 512 (2000).

107. D. GRASSO, E. TASSI, and F. L. WAELBROECK, Phys. Plasmas, 17, 082312 (2010).

108. F. L. WAELBROECK, R. D. HAZELTINE, and P. J. MORRISON, Phys. Plasmas, 16, 032109 (2009).

109. P. J. MORRISON, Phys. Plasmas, 12, 058102 (2005).

110. P. J. MORRISON, Rev. Mod. Phys., 70, 467 (1998).

111. F. L. WAELBROECK, P. J. MORRISON, and W. HORTON, Plasma Phys. Control. Fusion, 46, 1331 (2004).

112. P. J. MORRISON and J. M. GREENE, Phys. Rev. Lett., 45, 790 (1980); see also P. J. MORRISON and J. M. GREENE, Phys. Rev. Lett., Erratum: 48, 569 (1982).

113. E. TASSI, P. J. MORRISON, F. L. WAELBROECK, and D. GRASSO, Plasma Phys. Control. Fusion, 50, 085014 (2008).

114. P. J. MORRISON and M. KOTSCHENREUTHER, "The Free Energy Principle, Negative Energy Modes, and Stability," Nonlinear World: IV Int. Workshop on Nonlinear and Turbulent Processes in Physics, V. G. BARYAKHTAR, V. M. CHERNOUSENKO, N. S. EROKHIN, A. B. SITENKO, and V. E. ZAKHAROV, Eds., World Scientific (1990). 